Department of Economics and Finance

\author{
University of Guelph
}

Discussion Paper 2017-08

\title{
Strategic advance sales, demand uncertainty and overcommitment
}

\author{
By: \\ Sebastien Mitraille and Henry Thille
}




\title{
Strategic advance sales, demand uncertainty and overcommitment
}

\author{
Sébastien Mitraille* \\ Toulouse Business School \\ E-mail: s.mitraille@tbs-education.fr
}

\author{
Henry Thille \\ University of Guelph \\ E-mail: hthille@uoguelph.ca
}

November 17, 2017

\begin{abstract}
We study a game in which producers can sell in two periods: one before a random demand is fully revealed and one after. This type of game corresponds to models of strategic forward trading or of advance sales to intermediaries or consumers. Demand variations and committed advance sales results in the possibility that the net residual demand in the final stage may be so low that it is not profitable for producers make additional sales, or indeed, may even drive the final period price to zero, introducing some convexity into producers' payoffs. If this possibility of ex post overcommitment occurs on the equilibrium path, it reduces the level of advance sales chosen by producers, muting the pro-competitive effects found under deterministic demand. We establish a condition that determines whether or not demand uncertainty is "minor", in the sense that the equilibrium depends only on the expected value of the demand shock. In addition, we demonstrate that when the support of demand shocks is narrow enough compared to the marginal cost of production, there exists a unique symmetric subgame-perfect equilibrium in pure strategies. When the support of demand shocks is wider, we establish a regularity condition on the distribution of demand shocks and the model parameters that ensures the existence of a unique equilibrium in pure strategies. We illustrate through examples that commonly used uni-modal distributions satisfy this condition, while bi-modal distributions may not.
\end{abstract}

JEL Classification: C72, D43, L13

Keywords: Oligopoly, advance sales, uncertainty, overcommitment

Acknowledgement: The authors thank for their useful comments René Kirkegaard, Kurt Annen, Asha Sadanand, James Amegashie and seminar participants at the University of Guelph; Sergei Izmalkov, Rabah Amir, and participants of the Oligoworkshop 2016 (Université de Paris - Dauphine); Philippe Choné, Laurent Linnemer, Thibaud Vergé, and seminar participants at CREST-LEI; Simon Cowan and participants of the RES 2017 conference (University of Bristol).

\footnotetext{
${ }^{*}$ Corresponding author
} 


\section{Introduction}

The ability of firms to commit to sales in advance of market clearing creates a strategic incentive to capture future demand through these advance sales. This leads to market outcomes that are more competitive than what occurs when advance sales are prohibited since producers are unable to commit to not make additional sales in the future when the market clears. Examples include firms selling through forward contracts and firms selling to consumers or other intermediaries who then store for future consumption or resale. However, if the future demand is uncertain at the time the advance sales commitment is made, producers face the risk that they may have over-committed sales if demand turns out to be lower than expected. In this case, producers may find that further spot sales are unprofitable, in which case the strategic incentive for advance sales is likely to be muted as they have no effect on rivals spot sales in these subgames.

We analyze the effects of this possibility of ex-post overcommitment on the equilibrium of an advance sales game in which producers can commit to sell a quantity in a period prior to the meeting of the spot market and subsequently can produce additional output in the period in which the spot market meets. We find that the degree of uncertainty (as measured by the size of the support of the distribution of demand), as well as the shape of the density of demand shocks, have important consequences on the nature of the equilibrium and on its existence. This particular feature is due to the fact that in dynamic games like ours, in which producers can sell both before and after uncertainty is resolved, degenerate equilibria may occur in some sub-games, in the sense that given the realized shock and the strategies chosen in the past some or all producers may choose to not produce at all in the sub-game. Particular attention must then be paid to whether or not these subgame equilibria are on the equilibrium path. ${ }^{1}$ While it is possible that spot sales or price can be zero in our model, one contribution of this paper is to determine when these situations arise on the equilibrium path and to characterize the importance of this phenomenon for existence and nature of a pure strategy equilibrium.

We show that there are three qualitatively different types of equilibria: equilibria in which additional spot sales are made in every subgame (which we label "type I"); equilibria in which spot sales are not made in some subgames, but price is positive in every subgame ("type II"); and equilibria in which both spot sales and price are zero in some subgames ("type III"). We distinguish demand distributions with a narrow support, for which the equilibrium can only involve strictly positive spot prices (type I or II), from those with wide support for which the equilibrium can involve zero spot price as well (type III). Our main results are the following. In our first theorem, we establish that a unique pure-strategy equilibrium exists in the narrow support case for any distribution of demand shocks. When the lower bound on the support of the distribution of demand shocks is limited, spot sales are always strictly positive at equilibrium (type I) and demand uncertainty has no qualitative effect on the equilibrium in the sense that only the expected value of demand matters to oligopolists. In this sense, uncertainty is "minor" when the equilibrium is of type I. For more extreme lower bounds on the support of the distribution of demand shocks, spot sales can be nil in some subgames on equilibrium path and a type II equilibrium is the outcome: advance sales balance the usual strategic effects with the possibility that they are the sole source of profit obtained when demand is low.

In the wide support case, the possibility of a zero spot price results in the potential for payoffs to be convex. Our second theorem deals with this case, and establishes the existence of a unique pure-strategy equilibrium under a regularity assumption on the distribution of the stochastic component of demand and the other model primitives. ${ }^{2}$ This theorem establishes

\footnotetext{
${ }^{1}$ In a paper that generalizes the proof of existence and uniqueness of the classical static Cournot equilibrium to encompass the case of degenerate equilibria, Gaudet and Salant [11] point out, as a potential application, the importance of these equilibria when they occur in sub-games.

${ }^{2}$ Absent this regularity condition, multiple solutions to the first order condition of each firm may co-exist, and the best response in pure strategy may lack continuity. In the case where the regularity condition is not satisfied and the support of demand shocks is wide enough, the game still possesses an equilibrium in mixed strategy due
} 
that the condition under which the equilibrium involves only strictly positive spot sales (type I) is the same as in the narrow support case, so we can classify uncertainty 'minor' in the same way in the wide support case. When this condition is violated the equilibrium involves zero spot sales or even zero spot prices, i.e., is either type II or III. As our regularity assumption depends on marginal cost and the number of firms in addition to the demand distribution, neither the log-concavity of the density of demand shocks, nor an increasing hazard rate, is sufficient to satisfy the regularity assumption. ${ }^{3}$ We establish in a series of examples that bimodal distributions with modes located "close" to the extreme of the support of shocks fail to satisfy our regularity assumption, while common uni-modal distributions tend to satisfy it.

Our model can be seen as capturing the essential features of a number of different approaches that examine the incentives that producers have to sell to consumers in advance. One of these is the literature on strategic forward contracting following Allaz and Vila [2] (See Allaz [1], Thille [29], Ferreira [8], Mahenc and Salanié [19], Liski and Montero [17]) in which producers may enter binding forward contracts prior to the meeting of the spot market. In Allaz and Vila [2] since future demand is known with certainty, it does not matter whether the forward contracts are for cash settlement (the counter-parties simply exchange cash depending on the difference between the forward and spot prices) or physical delivery (the producer actually delivers the contracted quantity to the forward purchaser, who then resells on the spot market). While this distinction does not matter when spot demand is certain, when demand is uncertain this equivalence may break down as with physical delivery the committed production may turn out to be "too large" ex-post. ${ }^{4}$ Popescu and Seshadri [25] analyze a strategic forward trading model with stochastic demand drawn on a support unbounded from above, where the minimal inverse demand intercept can be as low as the unit cost of production. The importance of the demand support on the nature of equilibrium, as well as the consequences of the shape of the density of shocks on the existence of an individual best response, are not analyzed. In a model of supply function competition with costless production, Hölmberg and Willems [13] show that forward contracts in the form of an infinite series of options can be used by duopolists in equilibrium to commit their supply function on the spot market to have a negative slope, that is to commit to reduce competition. They establish this result in a costless production model, assuming that the hazard rate of the distribution of demand shocks either increases or is not too decreasing. In our model, where a single forward contract is used but production is costly, we show that existence of an equilibrium does not rely on assuming that the distribution of demand shocks has an increasing hazard rate (in the case where an assumption is needed).

A second approach to modelling strategic advance sales has producers selling physical production to other agents in a period prior to the spot market. Anton and Das Varma [3], Dudine, Hendel and Lizzeri [7], and Guo and Villas-Boas [12] study the effect of consumers storage on imperfect competition (respectively on a duopoly, on a monopoly, or in the case of differentiated products, but all in the absence of demand uncertainty). By increasing production in a period, producers cause a lower price which induces consumers to buy and store for a future period, lowering that future period's demand. Whereas a monopoly would prefer to let the price increase to limit consumer storage, a duopoly is better off encouraging consumers storage to compete more fiercely in the short run. In a similar vein, Mitraille and Thille [21, 22] study a model where speculators may purchase production in an earlier period, store the good, and then resell in the spot market ${ }^{5}$. Whereas demand uncertainty is one of the salient features of

to the continuity of the expected payoffs (See Fudenberg and Tirole [10])

${ }^{3}$ With the exception that in the case of costless production, an increasing hazard rate distribution satisfies our assumption and hence, is sufficient to guarantee the existence of an equilibrium.

${ }^{4}$ Allaz [1] allows for uncertainty in the strategic forward contracting game, but he does not allow for the possibility that firms may decide to produce no additional output for the spot market, or that the spot price might be driven to zero.

${ }^{5}$ Mitraille and Thille $[21,22]$ have multiple spot markets as well, but the forces at work are essentially the same. 
their model, they assume a limited capacity of storage or that small quantities can always be profitably produced by oligopolists, to rule out unprofitable spot sales from the equilibrium path. By inducing speculative purchases producers essentially are capturing future sales as the production sold to speculators returns as another source of supply in the future spot market. Our model can be viewed as capturing the essential features of these models: by selling in advance producers lower the net demand they face in the future.

In addition, our model is related to the analysis of static Cournot competition when quantities must be chosen before a random demand is known. Klemperer and Meyer [14] examine the choice between price and quantity competition in this setting, but essentially focus on 'minor' demand uncertainty only. However in the presence of larger uncertainty on the demand intercept, it is known that firms' individual payoffs can be a convex function of their sales, as well as those of their competitors. Lagerlöf [16] considers the problem of an oligopoly facing a linear demand with a random intercept which can take two values, high or low. He shows that multiple equilibria may be present due to the non-concavity induced by the possibility of a zero price occurring when demand is low. ${ }^{6}$ In our model, the possibility to sell once demand is revealed is endogenous, and enriches the set of equilibria while modifying the conditions required for the existence of an equilibrium: it introduces strategic considerations for selling in advance, ${ }^{7}$ but also provides oligopolists with an option to adjust sales by increasing them ex-post if a high enough demand stated is revealed.

The commitment to advance sales along with the possibility to make additional spot sales in our model is also reminiscent of the literature on the value of flexibility when uncertainty affects a commitment. ${ }^{8}$ In this literature, the timing of a single production period is endogenous, and production can happen either before or after uncertainty is realized. The focus is then on whether or not the first mover advantage survives the introduction of uncertainty, or on whether entry deterrence may occur. For example, Spencer and Brander [28] study the trade-off between production prior to the realization of uncertainty, where a firm gains Stackelberg leadership at the possible cost of overproduction, and production after the realization of uncertainty, in which a risk-free Cournot equilibrium obtains. In contrast, our approach supposes symmetry of moves and allows possible production in every period, so both ex-ante commitment and ex-post adaptation are possible. In this context we discuss the existence and the nature of subgame perfect Nash equilibria, which is not addressed in [28] and others.

We next present our model, followed by the determination of equilibrium in both the narrow and wide support cases. We then provide some examples of demand distributions and costs that both meet and fail our regularity condition.

\section{The Model}

Our model consists of two markets that meet in consecutive periods: an advance sales market in period one and a spot market in period two. We model the advance sales market as one in which producers make binding commitments to supply the product to competitive agents. ${ }^{9}$ These advance sales can be of two general types: first, physical production occurs in period one

\footnotetext{
${ }^{6}$ Lagerlöf [15] extends this analysis to consider a continuous distribution of demand shocks.

${ }^{7}$ In these papers, additional sales after demand is revealed are not possible (with the exception of the price competition case in Klemperer and Meyer [14]), so that there is no strategic effect of the advance sales.

${ }^{8}$ See Appelbaum and Lim [4], Perrakis and Warskett [24], Vives [31], Spencer and Brander [28], and Sadanand and Sadanand [26].

${ }^{9}$ Advance sales differ from advance production as defined in the models of Saloner [27] and Pal [23] in that in the former, the marginal revenue firms face when going on the "spot" market is residual one, while in the later firms are made more aggressive on the "spot" market due to the production realized they sell entirely when the market opens. In advance production games, the cost structure is crucial to the outcome of the game: equilibria can be asymmetric as a firm carrying a leader production is committed to sell it forcing its competitor to behave as a follower, but symmetric outcomes are also possible (Saloner [27]); cost differences between periods, such as discounting, then matters to equilibrium selection (Pal [23]).
} 
and the advance sales are delivered to the purchasers at that time, who then store at no cost for use in period two; and, second, forward contracts are entered in which settlement occurs by physical delivery of the contracted quantity ${ }^{10}$. Either way, net spot market demand faced by producers is reduced by the quantity of advance sales. Consumption occurs only in period two and is supplied by the sum of advance sales and any further production undertaken in period two.

There are $n$ producers, indexed by $i=1, \ldots, n$, who interact over two periods, $t=1,2$. At $t=1$, each producer may choose a quantity $x_{i}$ which is sold to competitive agents and has the effect of reducing net demand faced by producers at $t=2$. The price in the advance sale market is $p_{1}$. Let $\boldsymbol{x}=\left(x_{1}, x_{2}, \ldots, x_{n}\right), X=\sum_{i=1}^{n} x_{i}$, and $X_{-i}=\sum_{j \neq i} x_{j}$. In period two, each producer may choose to produce an additional quantity $y_{i}$ to sell on the spot market at a price $p_{2}$. We define $Y=\sum_{i=1}^{n} y_{i}$ and $Y_{-i}=\sum_{j \neq i} y_{j}$.

Firms face the same linear production costs in each period, ${ }^{11}$ so the total cost of production is given by $C\left(x_{i}, y_{i}\right)=c\left(x_{i}+y_{i}\right)$, with $c \geq 0$. Defining $p_{1}$ and $p_{2}$ as the price in each period, and assuming a discount factor equal to one,${ }^{12}$ the total profit for a producer is then

$$
\pi_{i}=\left(p_{1}-c\right) x_{i}+\left(p_{2}-c\right) y_{i} .
$$

Consumers' demand demand in the spot market is assumed to be linear in the market price $p_{2}$,

$$
D\left(p_{2} ; a\right)=\max \left\{a-p_{2}, 0\right\},
$$

where $a \in \mathbb{R}^{+}$is random with a continuous and differentiable cumulative distribution function $F(a)$ and density $f(a)$. It is realized at the beginning of period two and observed by all agents at that time. The support of $F(a)$ is the interval $\left[a_{L} ; a_{H}\right] \subseteq \mathbb{R}^{+}$, which is assumed to allow production and sales to be always potentially profitable, in the sense that if demand where known, some production would take place: ${ }^{13}$

$$
c \leq a_{L} \leq a_{H} \leq+\infty
$$

We assume that all agents observe the n-tuple of advance sales, ${ }^{14} \boldsymbol{x}$, as well as the realization of $a$ before they make their choices in period two. The inverse demand for the spot sales in period two is then

$$
P(Y, X, a)=\max \{a-(X+Y), 0\} .
$$

We assume free disposal if $X$ exceeds $a$.

\footnotetext{
${ }^{10}$ The counterparty to the forward contract receives the quantity specified in the forward contract and then resells it (if a "speculator") or consumes it.

${ }^{11}$ Linear production costs are necessary to be able to interpret the model as being consistent with both forward trading with physical delivery and competitive storage since splitting production across periods has no effect on cost. Convex production costs would give firms an additional cost-smoothing incentive to produce in the second period and would cloud the effect that we wish to analyze. In the same way, the constant marginal cost is the same in each period so that there is no inherent advantage in production costs between advance and spot sales.

${ }^{12} \mathrm{~A}$ unit discount factor means that the timing of the receipt of profits does not matter to a firm. This is important to be able to interpret $x_{i}$ as either advance sales made in period one (with revenue and costs realized in period one) or as forward contracts with physical delivery (with revenue and costs realized in period two).

${ }^{13}$ As such, we do not consider the risk that demand would be so low that firms would regret any production at all.

${ }^{14}$ Observability of advance sales matters in our setting, as it does more generally in all commitment games. Indeed the possibility of exerting some (endogenous) leadership through advance sales could be jeopardized if they are not observable. Bagwell [6] establishes that in a (textbook) Stackelberg game, non-observability of the leader's choice results in the Cournot outcome being the sole pure strategy equilibrium to the game. However Van Damme and Hurkens [30] demonstrate that a mixed strategy equilibrium close to the Stackelberg outcome exists in Bagwell's game, and Maggi [18] proves that private information restores the value of commitment. In the context of strategic forward trading, Ferreira [9] shows that imperfect observability can lead to multiple equilibria, including some that are even more competitive than in Allaz [2].
} 
Demand for advance sales in period one is derived from the expectation of $p_{2}$. The per-unit expected profit to an advance purchase is $E\left[p_{2} \mid X\right]-p_{1}$, where $E\left[p_{2} \mid X\right]$ is the expectation of $p_{2}$ conditional on the quantity of advance sales made. The competitive and rational agents purchasing the advance sales drive this expected profit to zero. Consequently, we have

$$
p_{1}=E\left[p_{2} \mid X\right],
$$

which represents the inverse demand faced by producers in the period one advance sales market.

From (1) it is clear that advance sales affect the marginal profit of spot sales only through the effect of aggregate advance sales on $p_{2}$. This allows us to restrict our attention to spot sales strategies that only depend on $X$ instead of the entire vector of individual advance sales, $\boldsymbol{x}$. A strategy for a firm consists of a pair $\omega_{i}=\left\{x_{i}, \tilde{y}_{i}(X, a)\right\}$ with $x_{i} \in \mathbb{R}_{+}$, and $\tilde{y}_{i}: \mathbb{R}_{+} \times\left[a_{L} ; a_{H}\right] \rightarrow$ $\mathbb{R}_{+}$. The spot sales strategy for a firm, $\tilde{y}_{i}(X, a)$, represents how the firm will play in subgames where total advance sales are $X$ and the state of demand is $a$. The expected payoff for any firm $i=1, \ldots, n$ given strategies for each producer is then

$$
E\left(\pi_{i}\left(\omega_{1}, \ldots, \omega_{n}\right)\right)=E\left[p_{2} \mid X\right] x_{i}+\int_{a_{L}}^{a_{H}} P\left(\sum_{i=1}^{n} \tilde{y}_{i}(X, a) ; X, a\right) \tilde{y}_{i}(X, a) d F(a)-c\left(x_{i}+\tilde{y}_{i}(X, a)\right) .
$$

We look for the subgame-perfect equilibria of this game, with $x_{i}^{*}$ and $y_{i}^{*}(X, a), i=1, \ldots n$, denoting the equilibrium strategies.

\section{Subgame equilibrium and expected profit}

We now establish the subgame equilibrium and expected payoff firms face when choosing their advance sales. We determine conditions on model parameters for which qualitatively different types of subgame equilibria are possible on the equilibrium path. In particular, we determine conditions under which there is a non-zero probability of zero spot sales and a non-zero probability of a zero spot price. This distinction matters as when these events occur off the equilibrium path, they do not affect firms' choices of advance sales, while when they occur on equilibrium path they must be taken into account. Once the correct expression of firms individual expected profit is obtained, the equilibrium to the game can be determined.

\subsection{Spot market equilibrium in period two}

At the beginning of period two, all agents observe the realization $a$, as well as $X$. Each firm solves

$$
\max _{y_{i} \geq 0}\left\{(P(Y, X, a)-c) y_{i}+\left(p_{1}-c\right) x_{i}\right\},
$$

where the second term represents the profits from advance sales which are sunk and have no effect on the choice of spot sales. The equilibrium spot sales in this subgame are straightforward to determine:

$$
y_{i}^{*}(X, a)=\max \left[\frac{a-X-c}{n+1}, 0\right], \quad i=1, \ldots n,
$$

which is the usual Cournot equilibrium adjusted for the reduction in demand due to the advance sales. The equilibrium spot price depends on whether or not spot sales are positive, but also must respect the non-negativity constraint on price: demand may turn out so low that it cannot accommodate the total advance sales. Consequently, we have

$$
p_{2}^{*}(X, a)= \begin{cases}0 & \text { if } a \leq X \\ a-X & \text { if } X \leq a \leq X+c \\ (a-X+n c) /(n+1) & \text { if } a>X+c\end{cases}
$$


with the first line representing a binding non-negativity constraint on price, and the second line representing a binding non-negativity constraint on spot sales. If neither non-negativity constraint binds, the price is simply the Cournot equilibrium price when demand is reduced by the total quantity of advance sales.

\subsection{Expected price and profits of the advance sales game}

In period one, agents make decisions based on their expectations over the outcomes in the period two subgames. Inverse demand for firms' advance sales is given by (5) and producers choose advance sales to optimize their expectation of profit, (1), with $y_{i}$ and $p_{2}$ replaced by (8) and (9). From (9) we see that the probability that period two price will be zero depends on $X$. If $X<a_{L}$, then $p_{2}>0$ in all subgames, while if $X \geq a_{L}$ there are subgames in which $p_{2}=0$. Consequently, a positive second period price occurs when $a>\max \left[a_{L}, X\right]$. Similarly, from (8) we see that if $X<a_{L}-c$, then $y_{i}>0$ in all subgames, while if $X \geq a_{L}-c$, there are subgames in which $y_{i}=0$. Consequently, positive spot sales occur for $a>\max \left[a_{L}, X+c\right]$. As rational agents use this information in forming expectations we have the following general expression for the expected price in period one,

$$
p_{1}=E\left[p_{2}^{*}(X, a) \mid X\right]=\int_{\max \left[a_{L}, X\right]}^{\max \left[a_{L}, X+c\right]}(a-X) d F(a)+\int_{\max \left[a_{L}, X+c\right]}^{a_{H}} \frac{a-X+n c}{n+1} d F(a) .
$$

As the qualitative nature of the equilibrium depends on whether or not a zero price or zero spot sales outcome is possible, we define the alternative types of equilibria as follows:

Definition 1 Given equilibrium advance sales of $X^{*}$, we say that the equilibrium is of

- Type I if $\operatorname{Pr}\left[p_{2}^{*}\left(X^{*}, a\right)=0\right]=0$ and $\operatorname{Pr}\left[y_{i}^{*}\left(X^{*}, a\right)=0\right]=0$,

- Type II if $\operatorname{Pr}\left[p_{2}^{*}\left(X^{*}, a\right)=0\right]=0$ and $\operatorname{Pr}\left[y_{i}^{*}\left(X^{*}, a\right)=0\right]>0$, and

- Type III if $\operatorname{Pr}\left[p_{2}^{*}\left(X^{*}, a\right)=0\right]>0$ and $\operatorname{Pr}\left[y_{i}^{*}\left(X^{*}, a\right)=0\right]>0$.

In a Type I equilibrium the non-negativity constraints for the spot price and spot sales do not bind for any possible realization of $a$. In a Type II equilibrium the non-negativity constraint for the spot price does not bind for any $a$ while there are some possible realizations of $a$ for which spot sales are zero. Finally, in a Type III equilibrium there are realizations of $a$ for which both spot price and sales are zero.

We can further refine our understanding of the possibility of a zero price by noting that all aggregate advance sales larger than $a_{H}-c$ are strictly dominated, as the price for the maximal demand would be below the marginal cost of production $c$ for all demand states, and hence will not be chosen by producers. This implies that we only need to consider $X \leq a_{H}-c$. Consequently, if $a_{L}>a_{H}-c$, the probability of a zero spot price is nil for any $X$ potentially played by producers, that is for any $X$ not strictly dominated. The equilibrium cannot be of Type III in this case. This observation motivates the following definition:

Definition $2 F(a)$ has narrow support if $a_{H}-a_{L} \leq c$ and has wide support if $a_{H}-a_{L}>c$.

If $F(a)$ has narrow support only equilibria of Type I or II are possible, while if $F(a)$ has wide support Type III equilibria must be considered. Clearly, if $c=0$ or $a_{H}=+\infty$ only the wide support case can occur.

We are now in a position to compute the expected profit for a firm in period one incorporating the equilibrium in the period two subgames, which we denote $\pi^{e}\left(x_{i}, X_{-i}\right)$. Using $y_{i}^{*}(X, a)$ and $p_{2}^{*}(X, a)$ from (8) and (9) in (1) we have

$$
\pi^{e}\left(x_{i}, X_{-i}\right)=E\left[\left(p_{2}^{*}\left(x_{i}+X_{-i}, a\right)-c\right)\left(x_{i}+y_{i}^{*}\left(x_{i}+X_{-i}, a\right)\right)\right] .
$$


The precise calculation of the expectation in (11) depends on whether $F(a)$ has narrow or wide support. In the case of a narrow support for $F(a)$ we have after some simplification ${ }^{15}$

$$
\pi^{e}\left(x_{i}, X_{-i}\right)= \begin{cases}\pi_{I}^{e}\left(x_{i}, X_{-i}\right) & \text { if } 0 \leq X \leq a_{L}-c \\ \pi_{I I}^{e}\left(x_{i}, X_{-i}\right) & \text { if } a_{L}-c<X \leq a_{H}-c .\end{cases}
$$

where

$$
\pi_{I}^{e}\left(x_{i}, X_{-i}\right)=\int_{a_{L}}^{a_{H}}\left(\frac{a-X-c}{n+1}\right)\left(x_{i}+\frac{a-X-c}{n+1}\right) d F(a)
$$

and

$$
\pi_{I I}^{e}\left(x_{i}, X_{-i}\right)=\int_{a_{L}}^{c+X}(a-X-c) x_{i} d F(a)+\int_{c+X}^{a_{H}}\left(\frac{a-X-c}{n+1}\right)\left(x_{i}+\frac{a-X-c}{n+1}\right) d F(a)
$$

When $F(a)$ has wide support, we have to account for the possibility of a zero price in period two when advance sales are relatively large. In this case we have

$$
\pi^{e}\left(x_{i}, X_{-i}\right)= \begin{cases}\pi_{I}^{e}\left(x_{i}, X_{-i}\right) & \text { if } 0 \leq X \leq a_{L}-c \\ \pi_{I I}^{e}\left(x_{i}, X_{-i}\right) & \text { if } a_{L}-c<X \leq a_{L} \\ \pi_{I I}^{e}\left(x_{i}, X_{-i}\right) & \text { if } a_{L}<X \leq a_{H}-c\end{cases}
$$

where $\pi_{I}^{e}\left(x_{i}, X_{-i}\right)$ and $\pi_{I I}^{e}\left(x_{i}, X_{-i}\right)$ are defined in (13) and (14) respectively, and

$$
\begin{aligned}
\pi_{I I I}^{e}\left(x_{i}, X_{-i}\right)=\int_{a_{L}}^{X}-c x_{i} d F(a)+\int_{X}^{c+X} & (a-X-c) x_{i} d F(a) \\
& +\int_{c+X}^{a_{H}}\left(\frac{a-X-c}{n+1}\right)\left(x_{i}+\frac{a-X-c}{n+1}\right) d F(a)
\end{aligned}
$$

We next determine the existence of a pure-strategy equilibrium to the game in the narrow and wide support cases respectively.

\section{Narrow support}

When $F(a)$ has narrow support it is possible to prove the existence of a unique symmetric equilibrium in pure strategies for any $F(a)$. We do this by demonstrating that i) expected profit is concave in $x_{i}$ given $X_{-i}$, and ii) the resulting unique best response is strictly decreasing and continuous.

Consider the case $x_{i} \leq a_{L}-c-X_{-i}$ in which the expected profit is given by $\pi_{I}^{e}\left(x_{i}, X_{-i}\right)$. The marginal effect of an increase in advance sales is given by

$$
\frac{\partial \pi_{I}^{e}\left(x_{i}, X_{-i}\right)}{\partial x_{i}}=\frac{(n-1)(E[a]-c)-2 n x_{i}-(n-1) X_{-i}}{(n+1)^{2}},
$$

which is strictly decreasing in $x_{i}$. This expression, which holds if advance sales are small enough, characterizes the expected marginal profit of selling in advance when it affects spot

\footnotetext{
${ }^{15}$ Specifically, from (9) we use

$$
p_{2}^{*}(X, a)-c= \begin{cases}-c & \text { if } a \leq X \\ a-X-c & \text { if } X \leq a \leq X+c \\ (a-X-c) /(n+1) & \text { if } a>X+c\end{cases}
$$
}


sales in all subgames, i.e., for all possible realizations of demand. ${ }^{16}$ This case corresponds to an equilibrium of Type I and if a symmetric equilibrium is of this type we denote $x_{I}^{*}$ the solution in $x$ of $\frac{\partial \pi_{I}^{e}(x,(n-1) x)}{\partial x_{i}}=0$, which can be determined in closed form as

$$
x_{I}^{*}=\frac{(n-1)(E[a]-c)}{n^{2}+1} .
$$

Next, consider $a_{L}-c-X_{-i}<x_{i} \leq a_{H}-c-X_{-i}$ for which $\pi_{I I}^{e}\left(x_{i}, X_{-i}\right)$ defines expected profit. Again, differentiating with respect to $x_{i}$ we obtain

$$
\begin{aligned}
\frac{\partial \pi_{I I}^{e}\left(x_{i}, X_{-i}\right)}{\partial x_{i}}= & \int_{a_{L}}^{c+X}\left(a-c-2 x_{i}-X_{-i}\right) d F(a)+\int_{c+X}^{a_{H}} \frac{(n-1)(a-c)-2 n x_{i}-(n-1) X_{-i}}{(n+1)^{2}} d F(a) \\
= & F(c+X)\left(E[a \mid a \leq X+c]-c-2 x_{i}-X_{-i}\right) \\
& +(1-F(c+X)) \frac{\left((n-1)(E[a \mid a \geq X+c]-c)-2 n x_{i}-(n-1) X_{-i}\right)}{(n+1)^{2}} .
\end{aligned}
$$

An equilibrium of this type is Type II and we denote $x_{I I}^{*}$ a solution in $x$ of $\frac{\partial \pi_{I I}^{e}(x,(n-1) x)}{\partial x_{i}}=0$, which cannot generally be determined in closed form. In this case, expected marginal profit is a weighted average of the effect on profit in subgames where no spot sales are made (the first term in (19)) and the effect on profit in subgames where spot sales are positive (the second term in (19)). The weights are the probabilities that subgames are reached for which $y^{*}(X, a)=0$, $F(c+X)$, and for which $y^{*}(X, a)>0,1-F(c+X)$. Marginal expected profit from advance sales corresponds to Cournot marginal profit in the subgames where $y^{*}(X, a)=0$ and to (17) in subgames where $y^{*}(X, a)>0$. So (19) represents a weighted average of Cournot behaviour on the advance sales market and of strategic advance sales behaviour with the weights being the probabilities of spot sales being zero and positive respectively. We see that the possibility of zero spot sales reduces the weight given to the strategic incentive to increase advance sales, suggesting that $x_{I I}^{*} \leq x_{I}^{*}$. We can now state the following theorem:

Theorem 1 When $F(a)$ has narrow support, there exists a unique symmetric equilibrium in pure strategies. The equilibrium individual advance sales level is given by

$$
\begin{aligned}
& \text { i) } \left.x_{I}^{*} \text { if } a_{L}>\hat{a} \text { (type } I\right) \text {, } \\
& \text { ii) } x_{I I}^{*} \text { if } a_{L} \leq \hat{a}(\text { type } I I) \text {, }
\end{aligned}
$$

with $\hat{a}=\frac{\left(n^{2}-n\right)}{n^{2}+1} E[a]+\frac{(n+1)}{n^{2}+1} c$.

Proof: See appendix.\|

Theorem 1i) demonstrates that for given values of $n$ and $c$, as long as the smallest possible realizations of $a$ are sufficiently high, equilibrium advance sales depend on the expected value of $a$ alone (no other characteristics of $F(a)$ matter). In this case, even if demand turns out to be the lowest possible, firms will still make additional spot sales in period two: $y_{i}^{*}\left(n x_{I}^{*}, a\right)>$ 0. Conversely, Theorem 1ii) demonstrates that when the lowest possible demand states are sufficiently low equilibrium advance sales take into account the possibility that spot sales may be unprofitable as reflected in the dependence of (19) on $E[a \mid a \leq X+c]$ and $F(X+c)$. The possibility that price falls below the marginal cost of production in period two forces firms to consider the effects of sub-games in which their sales come from advance sales only. In this case, equilibrium quantities are not solely based $E[a]$ but depend more generally on other characteristics of $F(a)$ as indicated in (19).

\footnotetext{
${ }^{16}$ This is the result corresponding to Allaz and Vila [2], with $E[a]$ substituted for the demand intercept.
} 
A striking feature of Theorem 1 is that the only assumption we have made regarding $F(a)$ is that it is continuous and differentiable, no other restriction on $F(a)$ is required. This is in contrast with the findings of Popescu and Seshadri [25] or Hölmberg and Willems [13], who obtain closely related uniqueness results by requiring the distribution of shocks to have an increasing hazard rate. However Popescu and Seshadri consider the wide support case only, while Holmberg and Willems impose costless production in the context of supply function competition, which is equivalent to require a wide support as we discussed above. ${ }^{17}$ This is also absent from the analysis by Mitraille and Thille [22], who study a model with a quadratic cost of production that effectively rules out the case of non-profitable production for spot sales. Moreover Theorem 1 also quantifies, in the context of the advance sales competition model we study, the notion of "minor" uncertainty for which, following conventional wisdom, the results of the literature are qualitatively unaffected by uncertainty. Uncertainty can be considered minor if the lower bound on the support of the distribution is not too low relative expected value: $a_{L}>\hat{a}$.

It is important to demonstrate that the condition under which a Type II equilibrium obtains, $a_{L}<\hat{a}$, can be satisfied for feasible values of the parameters, i.e., that it does not define an empty set in the parameter space. Since we assume $a_{L} \geq c$, part ii) of Theorem 1 is possible only if $c<\frac{\left(n^{2}-n\right)}{n^{2}+1} E[a]+\frac{(n+1)}{n^{2}+1} c$. As this is violated for $n=1$ a monopolist does not find itself in a situation where spot sales are unprofitable. Indeed, it is clear from (18) that a monopolist would not sell in advance at all. However, for $n>1$, this condition reduces to $c<E[a]$, which is true under our assumption that $c \leq a_{L}$. Consequently, the set of parameters for which a Type II equilibrium occurs is not empty for $n \geq 2,{ }^{18}$ and we have

Corollary 1 A Type II equilibrium does not occur for $n=1$, whereas for $n \geq 2$ there is a non-empty set of feasible parameters for which a Type II equilibrium obtains.

Furthermore, existence of a Type I equilibrium requires $a_{L}>\hat{a}=\frac{\left(n^{2}-n\right)}{n^{2}+1} E[a]+\frac{(n+1)}{n^{2}+1} c$. Whether or not this inequality can be satisfied depends on the model primitives. To see this, subtracting $c$ from both sides we have $a_{L}-c>\frac{n^{2}-n}{n^{2}+1}(E[a]-c)$. As $n$ increases, $\frac{n^{2}-n}{n^{2}+1}$ approaches one and the inequality cannot hold since $a_{L}<E[a]$. Furthermore, as $c$ approaches $a_{L}$ from below, $a_{L}-c$ approaches zero while $E[a]-c$ remains strictly positive, and again this inequality cannot hold. In summary we have

Corollary 2 The equilibrium may be is of Type I for $c$ and $n$ sufficiently small. For either $c$ large enough relative to $a_{L}$ or $n$ sufficiently large, the equilibrium can only be Type II.

The implication of Theorem 1 and Corollaries 1 and 2 is that the existing pro-competitive effects of advance sales identified in the literature may be tempered once uncertainty over future demand is allowed. Indeed, (19) indicates that $x_{I I}^{*}<x_{I}^{*}$. Since firms only care about the strategic effect of advance sales in subgames for which spot sales are positive, $x_{I I}^{*}$ lies between the Cournot level and $x_{I}^{*}$ when $1-F\left(X^{*}+c\right)<1$. While we have shown that equilibrium advance sales may be lowered by allowing for uncertain demand, it is instructive to investigate the magnitude of this effect. We do so by computing equilibrium advance sales for a particular example: a Uniform distribution for $a$ with support $[1-\delta, 1+\delta]$. In this case, $E[a]=1$ and we can control the type of equilibrium by varying $\delta$. To respect the assumption that $a_{L} \geq c$ we must have $\delta \leq 1-c$ and to have a narrow support requires $\delta \leq c / 2$, so we limit attention to $\delta \in(0, \min [c / 2,1-c])$. Setting $c=2 / 3, \delta \in(0,1 / 3)$ satisfies these restrictions. For the case $n=3$, we plot equilibrium advance sales relative to $x_{I}^{*}$ in Figure 1 to illustrate the proportional reduction in advance sales that occurs when the equilibrium is of Type II. A Type I equilibrium

\footnotetext{
${ }^{17}$ See Definition 2 and its discussion.

${ }^{18}$ Of course if we relax this assumption, allowing $a_{L}<c$, there are clearly demand states at which firms would not produce more in period two for any level of advance sales. In this case the equilibrium can only be of Type II.
} 
occurs in this example for $a_{L}=1-\delta \geq \hat{a}$, or $\delta \leq 1-\hat{a}=1-\frac{13}{15} \simeq 0.13$. Limited variations of demand around its mean does not change the equilibrium level of advance sales, and we see how $\hat{\delta} \equiv 1-\hat{a}$, illustrated as a vertical line, delineates an area in which the extent of uncertainty over demand is "minor" in that firms only consider $E[a]$ in their choices. However, for $\delta>\hat{\delta}$ the equilibrium is of Type II and advance sales are lower. For the largest value of $\delta$ consistent with a narrow support $(\delta=1 / 3)$, advance sales are nearly $40 \%$ lower than in a Type I equilibrium, demonstrating that the effect of the possibility of making zero spot sales in period two can be substantial.

\section{$5 \quad$ Wide support}

We turn now to the wide support case, $a_{H}-a_{L}>c$, allowing for the possibility of a Type III equilibrium with a non-zero probability of a zero price in period two. We demonstrate below that in this case, the expected profit may be convex in $x_{i}$ on the interval $\left[a_{L}-X_{-i}, a_{H}-c-X_{-i}\right]$. Continuity of the best response functions is therefore not guaranteed. ${ }^{19}$ Moreover, advance sales of a firm and of its competitors are not necessarily strategic substitutes in this case, allowing the possibility that best responses may not decline monotonically.

To obtain the existence of a unique continuous and decreasing best response function, and hence of a unique symmetric equilibrium, we add two conditions on the model primitives, which deal with the existence and slope of the best response respectively. The first condition states that if the expected profit is convex for some $x_{i} \in\left[a_{L}-X_{-i}, a_{H}-c-X_{-i}\right]$, the marginal expected profit is negative at these points. The second condition states that if the partial derivative of the marginal expected profit with respect to $X_{-i}$ is positive for some $x_{i} \in\left[a_{L}-\right.$ $\left.X_{-i}, a_{H}-c-X_{-i}\right]$, then the marginal expected profit is also negative at these points. That is under the conditions we introduce below, neither convexity issues of the expected profit, nor strategic complementarity of a firm's advance sales with its competitors', will interfere with the individual optimum of each firm; rather these issues will occur elsewhere in the domain of choices of each firm which is not part of an equilibrium, although not strictly dominated in the game-theoretical sense. ${ }^{20}$

\subsection{Equilibrium}

The marginal expected profit for $X<a_{L}$ has the same functional form as in the narrow support case, with the exception that the range over which (19) applies is reduced. In particular, (17) applies for $X \leq a_{L}-c$, while (19) applies for $a_{L}-c<X \leq a_{L}$. For $a_{L}<X \leq a_{H}-c$ marginal expected profit is given by

$$
\begin{aligned}
\frac{\partial \pi_{I I I}^{e}\left(x_{i}, X_{-i}\right)}{\partial x_{i}}=- & c F(X)+\int_{X}^{c+X}\left(a-c-2 x_{i}-X_{-i}\right) d F(a) \\
& +\int_{c+X}^{a_{H}} \frac{(n-1)(a-c)-2 n x_{i}-(n-1) X_{-i}}{(n+1)^{2}} d F(a) \\
=-c F(X) & +(F(c+X)-F(X))\left(E[a \mid X \leq a \leq X+c]-c-2 x_{i}-X_{-i}\right) \\
+ & (1-F(c+X)) \frac{(n-1)(E[a \mid a \geq X+c]-c)-2 n x_{i}-(n-1) X_{-i}}{(n+1)^{2}}
\end{aligned}
$$

which highlights the tradeoffs a firm makes when choosing advance sales. When increasing its advance sales, a firm increases the likelihood of a zero price in period two which is captured

\footnotetext{
${ }^{19}$ See also Lagerlöf $[15,16]$ in the context of static Cournot games with demand uncertainty.

${ }^{20}$ The additional condition we impose therefore implies that the expected profit is "single-peaked" in $x_{i}$ for every $X_{-i}$, i.e. is quasi-concave in $x_{i}$ for every $X_{-i}$. Mas-Colell, Whinston, and Green [20] proposition 8.D.3. (p. 253) establishes that normal form games with continuous and quasi-concave payoffs have pure strategy Nash equilibrium, and apply it to static models of oligopoly (section 12.C).
} 
by the first term in $(20)$. As $p_{1}^{*}=E\left[p_{2}^{*}(X, a) \mid X\right]$, this results in a lower price for advance sales since the advance purchasers face the risk that their purchase will be valueless in period two. This effect reduces further the incentive firms have to capture future demand through advance sales. An equilibrium in this case is of Type III and we denote by $x_{I I I}^{*}$ a solution in $x$ of $\frac{\partial \pi_{I I I}^{e}(x,(n-1) x)}{\partial x_{i}}=0$.

The existence of a unique pure strategy equilibrium of Type III is complicated by the potential for expected profit to be non-concave when $a_{L}<X \leq a_{H}-c$. Differentiating (20) with respect to $x_{i}$ and simplifying gives

$$
\begin{aligned}
\frac{\partial^{2} \pi_{I I I}^{e}\left(x_{i}, X_{-i}\right)}{\partial x_{i}^{2}}=x_{i} f(X)-\frac{n x_{i} f(c+X)}{n+1}-2(1-F(X)) & \\
+ & +\frac{2\left(n^{2}+n+1\right)}{(n+1)^{2}}(1-F(c+X))
\end{aligned}
$$

with the positive first and last terms potentially leading to a convex payoffs. We can express (21) as

$$
\frac{\partial^{2} \pi_{I I I}^{e}\left(x_{i}, X_{-i}\right)}{\partial x_{i}^{2}}=A(X) x_{i}+2 B(X)
$$

where

$$
A(X)=f(X)-\frac{n}{n+1} f(c+X)
$$

and

$$
B(X)=-(1-F(X))+\frac{n^{2}+n+1}{(n+1)^{2}}(1-F(c+X)) .
$$

We have $B(X)<0,{ }^{21}$ however $A(X)>0$ is possible, so concavity of $\pi_{I I I}^{e}$ in $x_{i}$ for a given $X_{-i}$ is not assured. Similarly, the derivative of the expected marginal profit with respect to $X_{-i}$ is

$$
\begin{aligned}
\frac{\partial^{2} \pi_{I I I}^{e}\left(x_{i}, X_{-i}\right)}{\partial x_{i} \partial X_{-i}}=x_{i} f(X)-\frac{n}{n+1} f(c+X) x_{i}-(1-F(X)) & \\
& +\frac{n^{2}+n+2}{(n+1)^{2}}(1-F(c+X)),
\end{aligned}
$$

which we write as

$$
\frac{\partial^{2} \pi_{I I I}^{e}\left(x_{i}, X_{-i}\right)}{\partial x_{i} \partial X_{-i}}=A(X) x_{i}+\tilde{B}(X)
$$

where

$$
\tilde{B}(X)=-(1-F(X))+\frac{n^{2}+n+2}{(n+1)^{2}}(1-F(c+X)) .
$$

While $\tilde{B}(X)<0,{ }^{22}$ marginal expected profit may be increasing in $X_{-i}$ as $A(X)>0$ cannot be ruled out, causing the possibility that best response functions may slope upward. Both of these potential problems for the existence of a unique pure-strategy equilibrium are due to the possibility that $A(X)>0$.

Nonetheless, these problems may be innocuous at the individual optimum of any firm, and hence at equilibrium, as long as the model parameters and the density $f(a)$ are regular enough. In order to state our condition we define two sets. First, the set of advance sales for a firm for which expected profit is concave in $x_{i}$ given $X_{-i}$ when $x_{i}+X_{-i}>a_{L}$ is

$$
S_{1}\left(X_{-i}\right) \equiv\left\{x_{i} \in\left[\max \left(a_{L}-X_{-i}, 0\right), a_{H}-c-X_{-i}\right]: A\left(x_{i}+X_{-i}\right) x_{i}+B\left(x_{i}+X_{-i}\right)<0\right\} .
$$

\footnotetext{
${ }^{21}$ Indeed $1-F(X) \geq 1-F(c+X)$ (with strict inequality for $\left.c>0\right)$, and $\left(n^{2}+n+1\right) /(n+1)^{2}<1$. Hence $(1-F(X))>\frac{n^{2}+n+1}{(n+1)^{2}}(1-F(X+c))$ and $B(X)<0$.

${ }^{22}$ The argument is similar to footnote 21 , with the exception that $\frac{n^{2}+n+2}{(n+1)^{2}} \leq 1$ for $n \geq 1$.
} 
Second, the set of advance sales for a firm for which marginal expected profit is decreasing in rivals' output in this case is

$$
S_{2}\left(X_{-i}\right) \equiv\left\{x_{i} \in\left[\max \left(a_{L}-X_{-i}, 0\right), a_{H}-c-X_{-i}\right]: A\left(x_{i}+X_{-i}\right) x_{i}+\tilde{B}\left(x_{i}+X_{-i}\right)<0\right\} .
$$

We make the following assumption:

Assumption 1 (Regularity) The model primitives, $c$, $n$, and $F(a)$, are such that for $X_{-i} \in$ $\left[0, a_{H}-c\right], S_{1}\left(X_{-i}\right)$ and $S_{2}\left(X_{-i}\right)$ are convex sets and, if non-empty, contain their lower bound, $\max \left(a_{L}-X_{-i}, 0\right)$.

Assumption 1 has the effect of limiting the number of changes in sign of $\partial^{2} \pi_{I I I}^{e} / \partial x_{i}^{2}$ and $\partial^{2} \pi_{I I I}^{e} / \partial x_{i} \partial X_{-i}$ as $x_{i}$ is varied for a given $X_{-i}$ to be at most one. The requirement that $S_{1}$ and $S_{2}$ contain their lower bound if non-empty implies that convexity of expected profit will only occur for relatively large values of individual, and hence aggregate, advance sales, i.e., for $x_{i}$ close to $a_{H}-c-X_{-i}$.

Assumption 1 imposes conditions on the number of firms, the marginal cost of production, and the distribution of demand shocks. Neither a distribution with an increasing hazard rate, nor more generally a log-concave distribution, necessarily satisfy it. ${ }^{23}$ To see this, if we denote $h(a)=f(a) /(1-F(a))$ as the hazard rate, we can write the second order derivatives of expected profit as

$$
\frac{\partial^{2} \pi_{I I I}^{e}\left(x_{i}, X_{-i}\right)}{\partial x_{i}^{2}}=f(X)\left(x_{i}-\frac{2}{h(X)}\right)-f(c+X)\left(\frac{n x_{i}}{n+1}-\frac{2\left(n^{2}+n+1\right)}{(n+1)^{2} h(X+c)}\right)
$$

and

$$
\frac{\partial^{2} \pi_{I I I}^{e}\left(x_{i}, X_{-i}\right)}{\partial x_{i} \partial X_{-i}}=f(X)\left(x_{i}-\frac{1}{h(X)}\right)-f(c+X)\left(\frac{n x_{i}}{n+1}-\frac{n^{2}+n+2}{(n+1)^{2} h(X+c)}\right),
$$

whose sign cannot be fixed by an assumption on the hazard rate alone, except in the particular case of costless production. When $c=0, f(X)$ can be factored in (30) and (31), and an increasing hazard rate $h(X)$ is sufficient to satisfy Assumption $1 .^{24}$

We can now state the following:

Theorem 2 When $F(a)$ has wide support, under Assumption 1 there exists a unique and symmetric equilibrium in pure strategies. Furthermore, the equilibrium is Type $I$ if $a_{L}>\hat{a}$ and either Type II or III otherwise, where $\hat{a}$ is as defined in Theorem 1.

Proof: See appendix.\|

The proof of Theorem 2 demonstrates that under Assumption 1 if a firm's best response to $X_{-i}$ results in $x_{i}+X_{-i}>a_{L}$ then it is in $S_{1}\left(X_{-i}\right)$ and $S_{2}\left(X_{-i}\right)$, i.e., expected profit is concave at the best response, which is itself decreasing in $X_{-i}$. The proof of Theorem 1 established that the expected profit is concave for any $x_{i}<a_{L}-X_{-i}$, so the assumption that $S_{1}\left(X_{-i}\right)$ contains $a_{L}-X_{-i}$ if it is non-empty implies that any non-concavity of expected profit occurs

\footnotetext{
${ }^{23}$ Bagnoli and Bergstrom [5] provide a detailed literature review of articles that use an increasing hazard rate or log-concavity assumption on the distribution of random preferences of buyers in order to establish the existence of pure-strategy equilibria. They show that log-concavity and and an increasing hazard rate are closely related: log-concavity of a PDF implies log-concavity of a CDF, which implies that the hazard rate of the distribution is increasing; however a distribution with an increasing hazard rate is not automatically log-concave.

${ }^{24}$ In this case, (30) is of the same sign as $\frac{x_{i}}{n+1}-\frac{2 n}{(n+1)^{2} h\left(x_{i}+X_{-i}\right)}$ and (31) is of the same sign as $\frac{x_{i}}{n+1}-$ $\frac{n-1}{(n+1)^{2} h\left(x_{i}+X_{-i}\right)}$. Both of these expressions are monotonically increasing in $x_{i}$ for a given $X_{-i}$ and so satisfy Assumption 1. See also Holmberg and Willems (2015).
} 
for large values of $x_{i}$. The largest value of $x_{i}$ considered by firms is $x_{i}=a_{H}-c-X_{-i}$ for which the marginal expected profit is negative. If the expected profit is convex at this point, then the marginal expected profit is increasing, which implies that the marginal expected profit is negative wherever the expected profit is convex and the existence of a pure strategy equilibrium is assured. The same argument is used to show that convexity of $S_{2}\left(X_{-i}\right)$ implies that strategic complementarity, which occurs when expected marginal profit increases with $X_{-i}$, can occur only where the expected marginal profit is negative i.e. only for advance sales in excess of the best response. Consequently, the best response function is continuous and decreasing which then implies uniqueness of the pure strategy equilibrium.

Theorem 2 establishes that the the definition of 'minor' uncertainty that was established in Theorem 1 remains the same in the wide support case. When $a_{L}>\hat{a}$, equilibrium advance sales and price depend only on $E[a]$ and are not affected by any other characteristics of $F(a)$.

For $a_{L}<\hat{a}$ the equilibrium is either of Type II or III. We can partially characterize which type obtains by demonstrating that a type III equilibrium obtains if $a_{L}$ and $c$ are sufficiently small:

Corollary 3 The equilibrium is of Type III if $a_{L}$ and $c$ are small enough.

Proof: See appendix.\|

We now extend our Uniform distribution example of the previous section to allow for a wide support. We have

$$
\frac{\partial^{2} \pi_{I I I}^{e}\left(x_{i}, X_{-i}\right)}{\partial x_{i}^{2}}=\frac{x_{i}}{(n+1)\left(a_{H}-a_{L}\right)}-\frac{2\left(a_{H}-x_{i}-X_{-i}\right)}{a_{H}-a_{L}}+\frac{2\left(n^{2}+n+1\right)}{(n+1)^{2}} \frac{a_{H}-x_{i}-X_{-i}-c}{a_{H}-a_{L}}
$$

from which we obtain

$$
S_{1}\left(X_{-i}\right)=\left\{x_{i} \in\left[\max \left(a_{L}-X_{-i}, 0\right), a_{H}-c-X_{-i}\right]: x_{i}<\frac{2\left(n\left(a_{H}-X_{-i}\right)+\left(n^{2}+n+1\right) c\right)}{3 n+1}\right\}
$$

which, if non-empty, is clearly a convex set containing its lower bound. A similar analysis can be done for the cross partial derivative. Using (25) we have

$$
\frac{\partial^{2} \pi_{I I I}^{e}\left(x_{i}, X_{-i}\right)}{\partial x_{i} \partial X_{-i}}=\frac{x_{i}}{(n+1)\left(a_{H}-a_{L}\right)}-\frac{a_{H}-x_{i}-X_{-i}}{a_{H}-a_{L}}+\frac{\left(n^{2}+n+2\right)}{(n+1)^{2}} \frac{a_{H}-x_{i}-X_{-i}-c}{a_{H}-a_{L}}
$$

from which we obtain

$S_{2}\left(X_{-i}\right)=\left\{x_{i} \in\left[\max \left(a_{L}-X_{-i}, 0\right), a_{H}-c-X_{-i}\right]: x_{i}<\frac{(n-1)\left(a_{H}-X_{-i}\right)+\left(n^{2}+n+2\right) c}{2 n}\right\}$

which is also clearly a convex set containing its lower bound if non-empty. Consequently, Assumption 1 is satisfied and Theorem 2 guarantees the existence of a unique pure-strategy equilibrium in this case for any $c<a_{H}-a_{L}$ and $n$. Figure 2 plots the equilibrium advance sales in this case and we see that as $\delta$ increases into the region of wide support advance sales continue to fall. The possibility of a zero price in period two reduces $p_{1}$, further reducing the incentive firms have to make advance sales.

The exponential distribution is another for which we can demonstrate analytically that Assumption 1 is satisfied for any $c$ and $n$. Restricting an exponential distribution to the support $[c,+\infty)$, i.e. $a_{L}=c>0$, its density is given by $f(a)=\lambda \exp \{-\lambda(a-c)\}$. It is straightforward to find

$S_{1}\left(X_{-i}\right)=\left\{x_{i} \in\left[\max \left(a_{L}-X_{-i}, 0\right), a_{H}-c-X_{-i}\right]: x_{i}<\frac{2}{\lambda} \frac{(n+1)^{2}-\left(n^{2}+n+1\right) \exp \{-\lambda c\}}{(n+1)(n+1-n \exp \{-\lambda c\})}\right\}$ 
and

$S_{2}\left(X_{-i}\right)=\left\{x_{i} \in\left[\max \left(a_{L}-X_{-i}, 0\right), a_{H}-c-X_{-i}\right]: x_{i}<\frac{1}{\lambda} \frac{(n+1)^{2}-\left(n^{2}+n+2\right) \exp \{-\lambda c\}}{(n+1)(n+1-n \exp \{-\lambda c\})}\right\}$

which are clearly convex sets containing their lower bounds if non-empty. Consequently Assumption 1 is satisfied and a unique pure-strategy equilibrium exists.

\section{$5.2 \quad$ Examples}

We now explore a number alternative distributions for $a$ in order to give a sense for the restrictiveness of Assumption 1. While we could determine analytically that the uniform and exponential distributions satisfied the assumption, this is not possible for most distributions, so we examine computed examples to further explore the restrictiveness of Assumption 1. For each example we plot the probability distribution function, the set $S_{1}\left(X_{-i}\right)$, and the best response of each firm to be show its intersection with the line $X_{-i} /(n-1)$ to which symmetric equilibria belong.

\subsubsection{Lognormal distribution}

The lognormal distribution has a non-monotonic hazard function and is not log-concave, so it is worth examining to see if it satisfies Assumption 1. We shift the lognormal distribution to $a_{L}=10$ and using a shape parameter of 1.0 we plot the density in Figure 3a). For parameters $n=3$, and $c=2$ we illustrate the situation in $\left(x_{i}, X_{-i}\right)$ space in Figure $\left.3 \mathrm{~b}\right)$. In this figure the region where $x_{i}+X_{-i}<a_{L}-c$ is labelled "I", $a_{L}-c<x_{i}+X_{-i}<a_{L}$ is labelled "II", and $a_{L}<x_{I}+X_{-i}<a_{H}-c$ is labelled "III". The region over which expected profit is convex is illustrated with blue dots. While there is a substantial area over which expected profit is convex, we see that Assumption 1 is satisfied: for a given $X_{-i}$ the values of $x_{i}$ for which the expected profit is concave forms a convex set. Expected profit is convex only for relatively large values of $x_{i}$. Theorem 2 then implies that a unique pure strategy equilibrium exists which we illustrate at the intersection of the best response and the line representing symmetric outputs, $x_{i}=X_{-i} /(n-1)$. The equilibrium in this case is of Type I.

This example demonstrates that Assumption 1 allows for the use of distributions that are ruled out by requiring an increasing hazard rate or a log-concave distribution.

\subsubsection{Uni-modal Beta distribution}

The next examples use the Beta distribution for $F(a)$ which has the advantage of taking on alternative shapes depending on its parameters. To start with a symmetric, uni-modal case we consider a Beta distribution with parameters $(1.5,1.5)$. We scale and shift this distribution so that its support is $[100,280]$ and plot it in Figure 4a). For $c=6$ and $n=3$, Figure $4 \mathrm{~b}$ ) shows that the situation is similar to the lognormal case above. While there is a large area where expected profit is convex, Assumption 1 is satisfied and a unique pure strategy exists, which is of Type III in this case. ${ }^{25}$

\subsubsection{U-shaped Beta distribution}

Next consider changing the parameters of the previous example to generate a bi-modal distribution. A Beta distribution with parameters $(0.5,0.5)$, i.e. an arc-sine distribution, has a U-shaped $f(a)$ as shown in the left of Figure 5. This example fails to satisfy Assumption 1, for low values of $X_{-i}$ expected profit has three isolated regions of concavity in $x_{i}$ and so $S_{1}\left(X_{-i}\right)$ is not convex. Although this part of the assumption is violated, the convexity in this case

\footnotetext{
${ }^{25}$ We have examined other uni-modal distributions, such as a Truncated Normal, with the same result.
} 
does not cause a problem for the computation of the best response. In addition, although not illustrated in Figure 5, $S_{2}\left(X_{-i}\right)$ is also not convex and we see that the best response function is not monotonically decreasing. The consequence for this example is the existence of multiple equilibria, one of Type II and one of Type III.

That this distribution causes problems is not surprising. As pointed out by Bagnoli and Bergstrom [5], the arc-sine probability distribution function is log-convex, and its hazard rate is U-shaped, so it also fails to satisfy those assumptions that have been made by other authors.

\subsubsection{Mixtures of truncated normal distributions}

The examples with a Beta distribution indicate bi-modal distributions may be particularly problematic for Assumption 1. To investigate this further we examine mixtures of truncated normal distributions ${ }^{26}$ to see the extent to which bi-modality causes problems. Consider an example where $F(a)$ is a mixture of truncated Normal distributions on [30;145], where the means are $\mu_{1}=80$ and $\mu_{2}=100$, standard deviation is $\sigma_{1}=\sigma_{2}=7$, and the mixing parameter is $\alpha=1 / 2$. The number of firms is $n=2$ and the marginal cost is $c=2$.

Results for this example are plotted in Figure 6 where we see that Assumption 1 is not satisfied for $X_{i}$ in a narrow range between roughly 50 and 60 . However, the best response function is well behaved and a unique pure strategy equilibrium is computed, illustrating that Assumption 1 is a sufficient but not necessary condition.

We next explore pulling the two modes apart for this example, setting the means to the extreme of the support, $\mu_{1}=a_{L}$ and $\mu_{2}=a_{H}$. This distribution is similar to the Beta $(0.5,0.5)$ example above in that the modes correspond to the extremes of the support of the distribution. We see from Figure 7 that $S_{1}\left(X_{-i}\right)$ is not convex for low values of $x_{-i}$, but this does not create problems for the existence of the best response in this case. However, $S_{2}\left(X_{-i}\right)$ also fails to be convex, in particular in the region around where the equilibrium occurs. This leads to the upward-sloping part of the best response, which in Figure 7 results in three equilibria, two of which have advance sales as the usual strategic substitutes, but one which occurs for advances sales which are strategic complements.

\section{Conclusion}

The ability to commit to sales in advance leads to pro-competitive strategic behaviour in a number of models in the literature. However, if demand is uncertain this strategic effect may be lessened depending on the significance of the demand uncertainty: if in low demand states firms do not wish to make additional sales, then the strategic incentive to make advance sales is lower. Allowing for the possibility of zero future spot sales or a zero future price can create problems for the curvature of firms' objective functions and consequently for the existence and uniqueness of an equilibrium in pure strategies. We have established the existence of pure-strategy equilibria in a game in which firms compete in advance for future demand, which corresponds to a number of different models in the literature. The nature of equilibrium depends on the significance of demand uncertainty as measured by the size of the support of the random demand component. We establish that a unique pure-strategy equilibrium exists as long as this support is sufficiently narrow. For wider supports, we establish a regularity condition that is sufficient for the existence of a unique pure-strategy equilibrium.

\footnotetext{
${ }^{26}$ The PDF of a truncated distribution on $\left[a_{L} ; a_{H}\right]$ with mean $\mu_{t}$ and standard deviation $\sigma_{t}$ is given by $\phi_{t}(a)=\frac{\frac{1}{\sqrt{2 \pi} \sigma_{t}} \exp \left\{-\left(a-\mu_{t}\right)^{2} / 2 \sigma_{t}^{2}\right\}}{\Phi\left(\left(a_{H}-\mu_{t}\right) / \sigma_{t}\right)-\Phi\left(\left(a_{L}-\mu_{t}\right) / \sigma_{t}\right)}$, where $\Phi(a)$ is the CDF of the standard Normal distribution. Truncation preserves log-concavity (see Bagnoli and Bergstrom, op.cit.), but mixture does not in the particular case of Normal distributions when means differ (available upon request). The PDF of the mixture of two truncated Normal distributions on $\left[a_{L}, a_{H}\right]$ is $f(a)=\alpha \phi_{1}(a)+(1-\alpha) \phi_{2}(a)$, where $\alpha \in[0,1]$.
} 
In determining the nature of the equilibrium we have identified a precise condition for which we can say uncertainty is "minor", i.e. for which the equilibrium is qualitatively similar to the one under certainty but with the random component of demand replaced by its expected value. This condition is independent of whether the support is narrow or wide, and if violated results in lower equilibrium advance sales. Hence the pro-competitive effects of advance sales are lessened under uncertainty if the uncertainty is significant enough.

We have also shown that problems for the existence of a pure strategy equilibrium only occur in the case of a distribution of demand shocks with a wide support. We presented a sufficient condition under which the potential convexity of payoffs does not cause a problem for the existence of a unique pure strategy equilibrium. This condition depends on the number of firms and marginal cost as well as the distribution of demand shocks, in contrast to previous papers that rely on an assumption of an increasing hazard rate. Indeed, we show that an increasing hazard rate is not sufficient to guarantee existence when there are positive marginal costs of production. Through the examination of a number of examples we find that bi-modal distributions of the demand uncertainty are the most likely to cause violations of our condition.

We have argued that our model conforms to a range of situations in which firms compete for future demand. These include selling in advance to consumers or speculators as well as forward trading. One difference in these different types of models is when production takes place. When selling in advance to consumers or speculators, production occurs when the sale is made which is prior to learning the state of demand. If demand turns out to be low so that price is below marginal cost, the outcome is ex post inefficient. However, if we interpret advance sales as forward contracting, production occurs after the realization of the demand state. In this case there is scope for renegotiation in the low demand states. For example, if demand is so low that price will be zero if all the contracted sales are produced, producers would be willing to pay up to their marginal cost to forsake delivering on their contracts and the purchasers of the advance sales would be willing to accept any payment in order to forgo delivery of the valueless good. In this case, the equivalence between cash settlement and physical delivery that is a characteristic of the strategic forward trading models of Allaz and Vila [2] and others breaks down.

While our results have been derived in a two-period model, we can conjecture some implications for the seminal result of Allaz and Vila [2] in which as the number of forward sales periods increases, so does the strategic effect, resulting in the perfectly competitive outcome in the limit. Our results suggest that this result is unlikely to be robust to allowing for demand uncertainty in the final spot market. As price approaches marginal cost, the probability that a firms will make zero spot sales due to a low demand state increases. This reduces the incentive to make the forward sales and suggests it is unlikely that producers will undertake sufficient advance sales to drive expected price to marginal cost.

The two-period assumption is clearly important in the wide support case in which there is a possibility of a zero second period price. The zero price is caused by advance sales exceeding a low demand state. However, in a longer horizon model it is less likely that this outcome occurs if the good is storable since agents may choose to store in the hopes of achieving a positive price in a future period. Consequently, we feel that extending the analysis to a longer time horizon is likely to reduce the likelihood of seeing a zero price in any period other than the final one, reducing the influence of the zero price possibility. However, we feel that the flavour of our results will not be affected: the combination of advance sales with the possibility of low demand states will reduce the incentive to use advance sales strategically due to the possibility that firms have "overcommitted" in some states of the world.

\section{References}

[1] Blaise Allaz. Oligopoly, uncertainty and strategic forward transactions. International Journal of Industrial Organization, 10(2):297-308, 1992. 
[2] Blaise Allaz and Jean-Luc Vila. Cournot competition, forward markets and efficiency. Journal of Economic Theory, 59(1):1-16, 1993.

[3] James J. Anton and Gopal Das Varma. Storability, market structure, and demand-shift incentives. The RAND Journal of Economics, 36(3):520-543, 2005.

[4] Elie Appelbaum and Chin Lim. Contestable markets under uncertainty. The RAND Journal of Economics, 16(1):28-40, 1985.

[5] Mark Bagnoli and Ted Bergstrom. Log-concave probability and its applications. Economic Theory, 26(2):445-469, 2005.

[6] Kyle Bagwell. Commitment and observability in games. Games and Economic Behavior, 8(2):271-280, 1995.

[7] Paolo Dudine, Igal Hendel, and Alessandro Lizzeri. Storable good monopoly: the role of commitment. American Economic Review, 96(5):1706-1719, 2006.

[8] José Luis Ferreira. Strategic interaction between futures and spot markets. Journal of Economic Theory, 108(1):141-151, 2003.

[9] José Luis Ferreira. The role of observability in futures markets. Topics in Theoretical Economics, 6(1):1-22, 2006.

[10] Drew Fudenberg and Jean Tirole. Game Theory. The MIT press, 1991.

[11] Gérard Gaudet and Stephen W. Salant. Uniqueness of Cournot equilibrium: New results from old methods. The Review of Economic Studies, 58(2):399, 1991.

[12] Liang Guo and J. Miguel Villas-Boas. Consumer stockpiling and price competition in differentiated markets. Journal of Economics \&3 Management Strategy, 16(4):827-858, 2007.

[13] Pär Holmberg and Bert Willems. Relaxing competition through speculation: Committing to a negative supply slope. Journal of Economic Theory, 159:236-266, 2015.

[14] Paul Klemperer and Margaret Meyer. Price competition vs. quantity competition: The role of uncertainty. The RAND Journal of Economics, 17(4):618-38, 1986.

[15] Johan N. M. Lagerlöf. Equilibrium uniqueness in a Cournot model with demand uncertainty. Topics in Theoretical Economics, 6(1):1-6, 2006.

[16] Johan N. M. Lagerlöf. Insisting on a non-negative price: Oligopoly, uncertainty, welfare, and multiple equilibria. International Journal of Industrial Organization, 25(4):861-875, 2007.

[17] Matti Liski and Juan-Pablo Montero. Forward trading and collusion in oligopoly. Journal of Economic Theory, 131(1):212-230, 2006.

[18] Giovanni Maggi. The value of commitment with imperfect observability and private information. The RAND Journal of Economics, pages 555-574, 1999.

[19] Philippe Mahenc and François Salanié. Softening competition through forward trading. Journal of Economic Theory, 116(2):282-293, 2004.

[20] Andreu Mas-Colell, Michael D. Whinston, and Jerry R. Green. Microeconomic Theory. Oxford University Press, New York, 1995.

[21] Sébastien Mitraille and Henry Thille. Monopoly behaviour with speculative storage. Journal of Economic Dynamics and Control, 33(7):1451-1468, 2009. 
[22] Sébastien Mitraille and Henry Thille. Speculative storage in imperfectly competitive markets. International Journal of Industrial Organization, 35:44-59, 2014.

[23] Debrashis Pal. Cournot duopoly with two production periods and cost differentials. Journal of Economic Theory, 55(2):441 - 448, 1991.

[24] Stylianos Perrakis and George Warskett. Capacity and entry under demand uncertainty. The Review of Economic Studies, 50(3):495-511, 1983.

[25] Dana G. Popescu and Sridhar Seshadri. Demand uncertainty and excess supply in commodity contracting. Management Science, 59(9):2135-2152, 2013.

[26] Asha Sadanand and Venkatraman Sadanand. Firm scale and the endogenous timing of entry: a choice between commitment and flexibility. Journal of Economic Theory, 70(2):516$530,1996$.

[27] Garth Saloner. Cournot duopoly with two production periods. Journal of Economic Theory, 42(1):183 - 187, 1987.

[28] Barbara J. Spencer and James A. Brander. Pre-commitment and flexibility. European Economic Review, 36(8):1601-1626, 1992.

[29] Henry Thille. Forward trading and storage in a Cournot duopoly. Journal of Economic Dynamics and Control, 27(4):651-665, 2003.

[30] Eric van Damme and Sjaak Hurkens. Games with imperfectly observable commitment. Games and Economic Behavior, 21(1):282-308, 1997.

[31] Xavier Vives. Technological competition, uncertainty, and oligopoly. Journal of Economic Theory, 48(2):386-415, 1989. 


\section{Appendix}

\section{Proof of Theorem 1}

The proof proceeds as follows. We first prove that there exists a unique best response, denoted $\hat{x}_{i}\left(X_{-i}\right)$, for each possible value of $X_{-i}$. Then we show that the best response $\hat{x}_{i}\left(X_{-i}\right)$ is continuous and everywhere strictly decreasing with respect to $X_{-i}$, with a slope smaller than 1 (in absolute value). Hence a unique symmetric equilibrium to the game can be determined by solving for $x^{*}$ in $x^{*}=\hat{x}_{i}\left((n-1) x^{*}\right)$.

Consider first the case $x_{i} \leq a_{L}-c-X_{-i}$. As (17) is clearly strictly decreasing in $x_{i}$, the expected profit is concave in $x_{i}$ given $X_{-i}$ on the interval $x_{i} \in\left[0, a_{L}-c-X_{-i}\right]$, and reaches a maximum either at

$$
x_{i}^{(1)}\left(X_{-i}\right)=\frac{(n-1)(E[a]-c)-(n-1) X_{-i}}{2 n}
$$

if this quantity is smaller than $a_{L}-c-X_{-i}$, that is if

$$
X_{-i}<\frac{2 n\left(a_{L}-c\right)-(n-1)(E[a]-c)}{n+1},
$$

or at $a_{L}-c-X_{-i}$ otherwise.

Consider now the case $x_{i} \in\left[a_{L}-c-X_{-i}, a_{H}-c-X_{-i}\right]$. From (19), at the upper bound of this interval, $x_{i}=a_{H}-c-X_{-i}$, marginal expected profit is strictly negative, ${ }^{27}$ and hence, if it exists, the advance sales that maximize expected profit must be strictly smaller than $a_{H}-c-X_{-i}$. Differentiating (19) with respect to $x_{i}$ yields

$$
\begin{aligned}
\frac{\partial^{2} \pi_{I I}^{e}\left(x_{i}, X_{-i}\right)}{\partial x_{i}^{2}}= & \left(c+X-c-2 x_{i}-X_{-i}\right) f(c+X)+\int_{a_{L}}^{c+X}-2 f(a) d a \\
& -\frac{(n-1) X-2 n x_{i}-(n-1) X_{-i}}{(n+1)^{2}} f(c+X)+\int_{c+X}^{a_{H}}-\frac{2 n}{(n+1)^{2}} f(a) d a \\
= & -x_{i} f(c+X)-2 F(c+X)+\frac{(n+1) x_{i} f(c+X)}{(n+1)^{2}}-\frac{2 n(1-F(c+X))}{(n+1)^{2}} \\
= & -\frac{n x_{i} f(c+X)}{n+1}-\frac{2 n}{(n+1)^{2}}-\frac{2\left(n^{2}+n+1\right) F(c+X)}{(n+1)^{2}}<0 .
\end{aligned}
$$

Expected profit is concave in $x_{i}$ given $X_{-i}$ on $x_{i} \in\left[a_{L}-c-X_{-i}, a_{H}-c-X_{-i}\right]$, and its maximum occurs either in the interior of $\left[a_{L}-c-X_{-i}, a_{H}-c-X_{-i}\right]$ or at its lower bound. We denote an interior maximum $x_{i}^{(2)}\left(X_{-i}\right)$ which is the unique $x_{i}$ that equates (19) to zero.

Expected marginal profit at $x_{i}=a_{L}-c-X_{-i}$ is obviously continuous. ${ }^{28}$ As it is strictly decreasing, checking that $x_{i}^{(2)}\left(X_{-i}\right)>a_{L}-c-X_{-i}$ is equivalent to verify whether $X_{-i}$ is such that the limit of the marginal profit (19) at $x_{i}=a_{L}-c-X_{-i}$ is strictly positive. It is immediate that $x_{i}^{(2)}\left(X_{-i}\right)>a_{L}-c-X_{-i}$ is equivalent to $X_{-i}>\frac{2 n\left(a_{L}-c\right)-(n-1)(E[a]-c)}{n+1}$, which is the condition on $X_{-i}$ under which the maximum of the expected profit on $\left[0, a_{L}-c-X_{-i}\right]$ is located at $x_{i}=a_{L}-c-X_{-i}$. Consequently, for $X_{-i}>\frac{2 n\left(a_{L}-c\right)-(n-1)(E[a]-c)}{n+1}$, marginal expected profit is positive and strictly decreasing on $\left[0, a_{L}-c-X_{-i}\right]$, strictly decreasing on $\left[a_{L}-c-X_{-i}, a_{H}-c-X_{-i}\right]$, and strictly negative at $x_{i}=a_{H}-c-X_{-i}$. Therefore expected profit is concave in $x_{i}$ for $x_{i} \in\left[0, a_{H}-c-X_{-i}\right]$ and there is a unique best response $\hat{x}_{i}\left(X_{-i}\right)$ which maximizes the expected profit of firm $i$ on the range $x_{i} \in\left[0, a_{H}-c-X_{-i}\right]$ when $a_{H}-a_{L} \leq c$, which is given by

$$
\hat{x}_{i}\left(X_{-i}\right)= \begin{cases}x_{i}^{(1)}\left(X_{-i}\right) & \text { if } X_{-i} \leq \frac{2 n\left(a_{L}-c\right)-(n-1)(E[a]-c)}{n+1} \\ x_{i}^{(2)}\left(X_{-i}\right) & \text { if } X_{-i}>\frac{2 n\left(a_{L}-c\right)-(n-1)(E[a]-c)}{n+1}\end{cases}
$$

\footnotetext{
${ }^{27}$ The first term in (19) is strictly negative while the second term is zero.

${ }^{28}$ Indeed, $X=a_{L}-c$ in this case and the first term in (19) is zero while the second is integrated between $a_{L}$ and $a_{H}$ which results in (17).
} 
We now demonstrate that each branch of the best response (41) is strictly decreasing in $X_{-i}$ with a slope smaller than 1 in absolute value. First, $x_{i}^{(1)}\left(X_{-i}\right)$ is a Cournot-like best response and is obviously strictly decreasing with respect to $X_{-i}$, with a slope smaller than 1 in absolute value. Let us establish it is also true for $x_{i}^{(2)}\left(X_{-i}\right)$.

The slope of this best response can be studied by applying the Implicit Function Theorem to the equation $\frac{\partial \pi_{I I}^{e}}{\partial x_{i}}=0$, which gives: $d x_{i}^{(2)} / d X_{-i}=-\frac{\partial^{2} \pi_{I I}^{e}}{\partial x_{i} \partial X_{-i}} / \frac{\partial^{2} \pi_{I I}^{e}}{\partial x_{i}^{2}}$. The cross partial derivative of the expected profit is given by:

$$
\begin{aligned}
\frac{\partial^{2} \pi_{I I}^{e}\left(x_{i}, X_{-i}\right)}{\partial x_{i} \partial X_{-i}}= & \left(c+X-c-2 x_{i}-X_{-i}\right) f(c+X)+\int_{a_{L}}^{c+X}(-1) f(a) d a \\
& -\frac{(n-1) X-2 n x_{i}-(n-1) X_{-i}}{(n+1)^{2}} f(c+X)+\int_{c+X}^{a_{H}}-\frac{n-1}{(n+1)^{2}} f(a) d a \\
= & -x_{i} f(c+X)-F(c+X)+\frac{(n+1) x_{i} f(c+X)}{(n+1)^{2}}-\frac{(n-1)(1-F(c+X))}{(n+1)^{2}} \\
= & -\frac{n x_{i} f(c+X)}{n+1}-\frac{n-1}{(n+1)^{2}}-\frac{\left(n^{2}+n+2\right) F(c+X)}{(n+1)^{2}}<0 .
\end{aligned}
$$

Therefore $x_{i}^{(2)}\left(X_{-i}\right)$ is strictly decreasing with respect to $X_{-i}$ and so is the best response $\hat{x}_{i}\left(X_{-i}\right)$. For the slope of the best response to be less than one in absolute value we require

$$
\begin{aligned}
& \left|\frac{\partial^{2} \pi_{I I}^{e}}{\partial x_{i} \partial X_{-i}}\right| /\left|\frac{\partial^{2} \pi_{I I}^{e}}{\partial x_{i}^{2}}\right|<1 \\
\Leftrightarrow & \frac{n-1}{(n+1)^{2}}+\frac{\left(n^{2}+n+2\right) F(c+X)}{(n+1)^{2}}<\frac{2 n}{(n+1)^{2}}+\frac{2\left(n^{2}+n+1\right) F(c+X)}{(n+1)^{2}} \\
\Leftrightarrow & \frac{n+1}{(n+1)^{2}}+\frac{\left(n^{2}+n\right) F(c+X)}{(n+1)^{2}}>0
\end{aligned}
$$

which holds.

The best response is obviously piecewise continuous. As the marginal expected profit is continuous at $X_{-i}=\frac{2 n\left(a_{L}-c\right)-(n-1)(E[a]-c)}{n+1}$, and decreasing in $X_{-i}$ below and above that threshold, the best response (41) is also continuous at $X_{-i}=\frac{2 n\left(a_{L}-c\right)-(n-1)(E[a]-c)}{n+1}$. Consequently there exists a unique and symmetric equilibrium to the game, given by the individual level of advance sales $x^{*}$ which solves $x^{*}=\hat{x}_{i}\left((n-1) x^{*}\right)$. In a Type I equilibrium $x_{I}^{*}=x_{i}^{(1)}\left((n-1) x_{I}^{*}\right)$ gives (18) which must satisfy

$$
(n-1) x_{I}^{*}<\frac{2 n\left(a_{L}-c\right)-(n-1)(E[a]-c)}{n+1} .
$$

This is equivalent, after substituting for $x_{I}^{*}$, to

$$
a_{L}>\frac{n^{2}-n}{n^{2}+1} E[a]+\frac{n+1}{n^{2}+1} c \equiv \hat{a} .
$$

Then for $a_{L} \leq \hat{a}$ the equilibrium is of Type II, where the individual level of advance sales $x_{I I}^{*}$ is the unique solution in $x$ of $\frac{\partial \pi_{I I}^{e}(x,(n-1) x)}{\partial x_{i}}=0$.

\section{Proof of Theorem 2}

The proof proceeds as the proof of Theorem 1. First, we prove that there exists a best response, denoted $\hat{x}_{i}\left(X_{-i}\right)$, which is unique and continuous for each possible value of $X_{-i}$. Contrary to the case of a narrow support of demand shocks, the expected marginal profit is convex when $X$ is large enough in the range of non-dominated advance sales. Assumption 1 then insures that 
this feature does not jeopardize the existence of a unique best response, by guaranteeing the expected profit is convex only when the expected marginal profit is negative, i.e. for individual quantities a firm would never play in response to $X_{-i}$. Once the best response $\hat{x}_{i}\left(X_{-i}\right)$ is characterized, we prove that it is everywhere strictly decreasing with respect to $X_{-i}$ with a slope smaller than 1 (in absolute value). Then a symmetric equilibrium to the game can be determined by solving for $x^{*}$ in $x^{*}=\hat{x}_{i}\left((n-1) x^{*}\right)$, which is unique.

Let us start with the characterization of the best response. As the expression of the expected profit (15) shows, we must be concerned with three different forms of the marginal profit, depending whether $x_{i}+X_{-i} \leq a_{L}-c$, or whether $x_{i}+X_{-i} \in\left[a_{L}-c ; a_{L}\right]$, or last whether $x_{i}+X_{-i} \in\left[a_{L} ; a_{H}-c\right]$. We have already established in the proof of Theorem 1 that the expected profit reaches a maximum at $x^{(1)}\left(X_{-i}\right)$ if $X_{-i} \leq \frac{2 n\left(a_{L}-c\right)-(n-1)(E[a]-c)}{n+1}$, and at $x^{(2)}\left(X_{-i}\right)$ if $X_{-i}>\frac{2 n\left(a_{L}-c\right)-(n-1)(E[a]-c)}{n+1}$, with a marginal profit strictly decreasing in $x_{i}$ and continuous along $x_{i}+X_{-i}=a_{L}-c$. In the wide support case we are currently considering, we must also check whether $x^{(2)}\left(X_{-i}\right)$ belongs to the set of values of $X$ on which $\pi_{I I}^{e}\left(x_{i}, X_{-i}\right)$ is defined, i.e. $x^{(2)}\left(X_{-i}\right)<a_{L}-X_{-i}$, and study the maxima of the expected profit when it is not the case.

As the marginal profit $\frac{\partial \pi_{I I}^{e}\left(x_{i}, X_{-i}\right)}{\partial x_{i}}$ is strictly decreasing in $x_{i}$ given $X_{-i}$, checking whether $x^{(2)}\left(X_{-i}\right)<a_{L}-X_{-i}$ is equivalent to verify that $X_{-i}$ is such that $\frac{\partial \pi_{I I}^{e}\left(a_{L}-X_{-i}, X_{-i}\right)}{\partial x_{i}}<0$. We have

$$
\begin{aligned}
& \frac{\partial \pi_{I I}^{e}\left(a_{L}-X_{-i}, X_{-i}\right)}{\partial x_{i}}=F\left(a_{L}+c\right)\left(E\left(a \mid a \leq a_{L}+c\right)-c\right) \\
& +\frac{(n-1)\left(1-F\left(a_{L}+c\right)\right)}{(n+1)^{2}}\left(E\left(a \mid a \geq a_{L}+c\right)-c\right)-2\left(F\left(a_{L}+c\right)+\frac{n\left(1-F\left(a_{L}+c\right)\right)}{(n+1)^{2}}\right) a_{L} \\
& +\left(F\left(a_{L}+c\right)+\frac{1-F\left(a_{L}+c\right)}{n+1}\right) X_{-i}
\end{aligned}
$$

which is linear in $X_{-i}$, and hence is negative if

$$
X_{-i} \leq \frac{N\left(a_{L}\right)}{D\left(a_{L}\right)}
$$

where

$$
\begin{array}{r}
N\left(a_{L}\right)=2\left(\frac{\left((n+1)^{2}-n\right) F\left(a_{L}+c\right)+n}{(n+1)^{2}}\right) a_{L}-F\left(a_{L}+c\right)\left(E\left(a \mid a \leq a_{L}+c\right)-c\right) \\
-\frac{(n-1)}{(n+1)^{2}}\left(1-F\left(a_{L}+c\right)\right)\left(E\left(a \mid a \geq a_{L}+c\right)-c\right),
\end{array}
$$

and

$$
D\left(a_{L}\right)=\frac{n F\left(a_{L}+c\right)+1}{n+1} .
$$

Due to the fact that the expected marginal profit is continuous and decreasing in $X_{-i}$ in cases I and II, we have $\frac{N\left(a_{L}\right)}{D\left(a_{L}\right)}>\frac{2 n\left(a_{L}-c\right)-(n-1)(E[a]-c)}{n+1}$. If $X_{-i}>\frac{N\left(a_{L}\right)}{D\left(a_{L}\right)}$, then, as the marginal profit is decreasing for all values of $x_{i}$ lower than $a_{L}-X_{-i}$, the maximum of the expected profit on $\left[0, a_{L}-c-X_{-i}\right] \cup\left[a_{L}-c-X_{-i}, a_{L}-X_{-i}\right]$ is at $x_{i}=a_{L}-X_{-i}$, in which case the optimum occurs in $\left[a_{L}-X_{-i}, a_{H}-c-X_{-i}\right]$.

For $x_{i} \in\left[a_{L}-X_{-i}, a_{H}-c-X_{-i}\right]$ the expected marginal profit is given by (20), which takes into account the probability of a zero price and can be expressed as

$$
\begin{aligned}
\frac{\partial \pi_{I I I}^{e}\left(x_{i}, X_{-i}\right)}{\partial x_{i}}= & -c F(X)+(F(c+X)-F(X))\left(E(a \mid X \leq a \leq c+X)-c-2 x_{i}-X_{-i}\right) \\
& +(1-F(c+X)) \frac{(n-1)(E(a \mid a \geq c+X)-c)-2 n x_{i}-(n-1) X_{-i}}{(n+1)^{2}} .
\end{aligned}
$$


First, expected marginal profit is continuous at $x_{i}=a_{L}-X_{-i}$ :

$$
\begin{gathered}
\frac{\partial \pi_{I I I}^{e}\left(a_{L}-X_{-i}, X_{-i}\right)}{\partial x_{i}}=F\left(c+a_{L}\right)\left(E\left(a \mid a_{L} \leq a \leq c+a_{L}\right)-c-2\left(a_{L}-X_{-i}\right)-X_{-i}\right) \\
+\left(1-F\left(c+a_{L}\right)\right) \frac{(n-1)\left(E\left(a \mid a \geq c+a_{L}\right)-c\right)-2 n\left(a_{L}-X_{-i}\right)-(n-1) X_{-i}}{(n+1)^{2}} \\
=F\left(c+a_{L}\right)\left(E\left(a \mid a_{L} \leq a \leq c+a_{L}\right)-c\right)+\left(1-F\left(c+a_{L}\right)\right) \frac{(n-1)\left(E\left(a \mid a \geq c+a_{L}\right)-c\right)}{(n-1)^{2}} \\
-2\left(F\left(a_{L}+c\right)+\frac{n}{(n+1)^{2}}\left(1-F\left(c+a_{L}\right)\right)\right) a_{L}-\left(F\left(a_{L}+c\right)+\frac{1-F\left(c+a_{L}\right)}{n+1}\right) X_{-i} \\
=\frac{\partial \pi_{I I}^{e}\left(a_{L}-X_{-i}, X_{-i}\right)}{\partial x_{i}}
\end{gathered}
$$

However, expected marginal profit is not always decreasing in the range $x_{i} \in\left[a_{L}-X_{-i}, a_{H}-\right.$ $\left.c-X_{-i}\right]$. Indeed, the derivative of expected marginal profit, (21), changes sign at most once in the range $x_{i} \in\left[a_{L}-X_{-i}, a_{H}-c-X_{-i}\right]$ under Assumption 1, from negative to positive values. Therefore under assumption 1, two situations must be distinguished: the case (a) where, given $X_{-i}$, expected marginal profit is strictly increasing in $x_{i}$ for $x_{i} \in\left[a_{L}-X_{-i}, a_{H}-c-X_{-i}\right]$, from the case (b) where, given $X_{-i}$, there exists $\bar{x}\left(X_{-i}\right) \in\left(a_{L}-X_{-i}, a_{H}-c-X_{-i}\right)$ such that marginal expected profit is decreasing for $x_{i} \leq \bar{x}\left(X_{-i}\right)$ and increasing for $x_{i}>\bar{x}\left(X_{-i}\right)$. Before analyzing these two cases, note that at the upper bound, $x_{i}=a_{H}-c-X_{-i}$, the total amount of advance sales $X$ is equal to $a_{H}-c$, so that $c+X=a_{H}$, and the expected marginal profit is equal to

$$
\begin{aligned}
\frac{\partial \pi_{I I I}^{e}\left(a_{H}-c-X_{-i}, X_{-i}\right)}{\partial x_{i}} & =-c F\left(a_{H}-c\right) \\
+ & \left(1-F\left(a_{H}-c\right)\right)\left(E\left(a \mid a_{H}-c \leq a \leq a_{H}\right)-2 a_{H}+c+X_{-i}\right),
\end{aligned}
$$

which is strictly negative for non-dominated advance sales $x_{i}$. Indeed, as $E\left(a \mid a_{H}-c \leq a \leq\right.$ $\left.a_{H}\right) \leq a_{H}$

$$
E\left(a \mid a_{H}-c \leq a \leq a_{H}\right)-2 a_{H}+c+X_{-i} \leq-a_{H}+c+X_{-i} \leq 0
$$

as we are considering non-dominated levels of advance sales, $X_{-i} \leq a_{H}-c$. Note also that when the support is unbounded, $a_{H}=+\infty$, then the limit of the marginal expected profit above is equal to $-c<0$; hence our analysis also applies to the case where the support of demand shocks is unbounded from above. Consequently in case (a), marginal expected profit is strictly increasing in $x_{i}$ to a negative value at $x_{i}=a_{H}-c-X_{-i}$; therefore, marginal expected profit is strictly negative for all values of $x_{i} \in\left[a_{L}-X_{-i}, a_{H}-c-X_{-i}\right]$. On the other hand in case (b), negative marginal expected profit at $x_{i}=a H-c-X_{-i}$ implies that it is also negative at $\bar{x}\left(X_{-i}\right)$ and so is negative whenever it is increasing. We can now complete the determination of the global optimum of the expected profit.

In the case where $X_{-i} \leq \frac{N\left(a_{L}\right)}{D\left(a_{L}\right)}$ analyzed above, marginal expected profit is negative at $x_{i}=a_{L}-X_{-i}$. Since it is continuous, then either it is strictly increasing for $x_{i} \in\left[a_{L}-\right.$ $\left.X_{-i}, a_{H}-c-X_{-i}\right]$ but always remains negative (case (a)), or is decreasing and then increasing again for $x_{i} \in\left[a_{L}-X_{-i}, a_{H}-c-X_{-i}\right]$, but remains again always negative. In both cases the global optimum of the expected profit is $x^{(2)}\left(X_{-i}\right)$.

On the contrary when $X_{-i}>\frac{N\left(a_{L}\right)}{D\left(a_{L}\right)}$, expected marginal profit is by continuity positive at the right of $x_{i}=a_{L}-X_{-i}$. As it is negative at $x_{i}=a_{H}-c-X_{-i}$, it must decrease as long as $x_{i}$ is small enough in $\left[a_{L}-X_{-i}, a_{H}-c-X_{-i}\right]$, to become negative and then increase again to $x_{i}=a_{H}-c-X_{-i}$, where it is negative. Consequently a unique maximum to the expected 
profit must exist in $\left[a_{L}-X_{-i}, a_{H}-c-X_{-i}\right]$, denoted $x_{i}^{(3)}\left(X_{-i}\right)$ which is the solution in $x_{i}$ of

$$
\begin{aligned}
0= & -c F\left(x_{i}+X_{-i}\right) \\
+ & \left(F\left(c+x_{i}+X_{-i}\right)-F\left(x_{i}+X_{-i}\right)\right)\left(E\left(a \mid x_{i}+X_{-i} \leq a \leq c+x_{i}+X_{-i}\right)-c-2 x_{i}-X_{-i}\right) \\
& +\left(1-F\left(c+x_{i}+X_{-i}\right)\right) \frac{(n-1)\left(E\left(a \mid a \geq c+x_{i}+X_{-i}\right)-c\right)-2 n x_{i}-(n-1) X_{-i}}{(n+1)^{2}} .
\end{aligned}
$$

To summarize, we have demonstrated that the best response exists and is given by

$$
\hat{x}_{i}\left(X_{-i}\right)= \begin{cases}x_{i}^{(1)}\left(X_{-i}\right) & \text { if } X_{-i} \leq \frac{\left(2 n\left(a_{L}-c\right)-(n-1)(E[a]-c)\right)}{n+1} \\ x_{i}^{(2)}\left(X_{-i}\right) & \text { if } \frac{\left(2 n\left(a_{L}-c\right)-(n-1)(E[a]-c)\right)}{n+1}<X_{-i} \leq \frac{N\left(a_{L}\right)}{D\left(a_{L}\right)} \\ x_{i}^{(3)}\left(X_{-i}\right) & \text { if } X_{-i}>\frac{N\left(a_{L}\right)}{D\left(a_{L}\right)} .\end{cases}
$$

The second step of the proof consists in demonstrating that the the best response defined above is continuous and strictly decreasing. First, it is continuous at $X_{-i}=\frac{\left(2 n\left(a_{L}-c\right)-(n-1)(E[a]-c)\right)}{n+1}$ as we demonstrated in the proof of Theorem 1. The same reasoning allows to prove that the best response is also continuous at $X_{-i}=\frac{N\left(a_{L}\right)}{D\left(a_{L}\right)} \equiv \tilde{X}$. First note that at $\left(x^{(2)}(\tilde{X}), \tilde{X}\right)$, $x^{(2)}(\tilde{X})+\tilde{X}=a_{L}$ and $\frac{\partial \pi_{I I}^{e}\left(x^{(2)}(\tilde{X}), \tilde{X}\right)}{\partial x_{i}}=0$. Then as the expected marginal profit is strictly decreasing in $x_{i}$ at the best response, if $\frac{\partial \pi_{I I I}^{e}\left(x^{(2)}(\tilde{X}), \tilde{X}\right)}{\partial x_{i}}=0, x^{(3)}(\tilde{X})=x^{(2)}(\tilde{X})$ and the best response is continuous at $\tilde{X}$. Using its definition, we can compute

$$
\begin{aligned}
\frac{\partial \pi_{I I I}^{e}\left(x_{i}^{(2)}(\tilde{X}), \tilde{X}\right)}{\partial x_{i}} & =-c F\left(x^{(2)}(\tilde{X})+\tilde{X}\right)+\int_{x^{(2)}(\tilde{X})+\tilde{X}}^{c+x^{(2)}(\tilde{X})+\tilde{X}}\left(a-c-2 x^{(2)}(\tilde{X})-\tilde{X}\right) d F(a) \\
+ & \int_{c+x^{(2)}(\tilde{X})+\tilde{X}}^{a_{H}} \frac{(n-1)(a-c)-2 n x^{(2)}(\tilde{X})-(n-1) \tilde{X}}{(n+1)^{2}} d F(a) \\
& =-c F\left(a_{L}\right)+\int_{a_{L}}^{c+a_{L}}\left(a-c-2 x^{(2)}(\tilde{X})-\tilde{X}\right) d F(a) \\
& +\int_{c+a_{L}}^{a_{H}} \frac{(n-1)(a-c)-2 n x^{(2)}(\tilde{X})-(n-1) \tilde{X}}{(n+1)^{2}} d F(a) \\
& =\frac{\partial \pi_{I I}^{e}\left(x_{i}^{(2)}(\tilde{X}), \tilde{X}\right)}{\partial x_{i}}=0 .
\end{aligned}
$$

Therefore under Assumption 1, the best response must be continuous at $X_{-i}=\frac{\left(2 n\left(a_{L}-c\right)-(n-1)(E[a]-c)\right)}{n+1}$ and at $X_{-i}=\tilde{X}$.

We can now determine the slope of $\hat{x}^{i}\left(X_{-i}\right)$. For the first two cases in (55) it is strictly decreasing as already demonstrated in the proof of Theorem 1. For the third case, applying the Implicit Function Theorem to $x_{i}^{(3)}\left(X_{-i}\right)$, we have:

$$
\frac{d x_{i}^{(3)}}{d X_{-i}}=-\frac{\frac{\partial^{2} \pi_{I I x_{i}}^{e}\left(x_{i}, X_{-i}\right)}{\partial X_{-i}}}{\frac{\partial^{2} \pi_{I I I}^{e}\left(x_{i}, X_{-i}\right)}{\partial\left(x_{i}\right)^{2}}}
$$

with the numerator given by (21) and the denominator by (25). We have already established that the denominator is negative at the best response: indeed, $x^{(3)}\left(X_{-i}\right)$ must belong to the set of values $S_{1}\left(X_{-i}\right)$ in which the second derivative of the expected profit with respect to $x_{i}$ is negative.

To establish the sign of the cross-partial derivative, note that the marginal expected profit at $X_{-i}=N\left(a_{L}\right) / D\left(a_{L}\right)$ must be positive, otherwise the best response would not be $x_{i}^{(3)}\left(X_{-i}\right)$. 
To sign the denominator, consider levels of advance sales such that $x_{i}+X_{-i}=a_{H}-c$. Clearly, from (50), marginal expected profit is negative at that level of advance sales. From (25) we have

$$
\left.\frac{\partial^{2} \pi_{I I I}^{e}\left(x_{i}, X_{-i}\right)}{\partial x_{i} \partial X_{-i}}\right|_{X=a_{H}-c}=x_{i} f\left(a_{H}-c\right)-\frac{n}{n+1} f\left(a_{H}\right)-\left(1-F\left(a_{H}-c\right)\right),
$$

the sign of which can be negative or positive. If negative, Assumption 1 implies that $\frac{\partial^{2} \pi_{I I}^{e}}{\partial x_{i} \partial X_{-i}}<0$ for all non-dominated levels of advance sales. If positive, since $\frac{\partial \pi_{I I I}^{e}}{\partial x_{i}}<0$ at $x_{i}+X_{-i}=a_{H}-c$, Assumption 1 implies that $\frac{\partial \pi_{I I}^{e}}{\partial x_{i}}<0$ wherever $\frac{\partial^{2} \pi_{I I}^{e}}{\partial x_{i} \partial X_{-i}}>0$, so $\frac{\partial^{2} \pi_{I I I}^{e}}{\partial x_{i} \partial X_{-i}}<0$ at $x_{i}^{(3)}\left(X_{-i}\right)$. Consequently the best response $x_{i}^{(3)}\left(X_{-i}\right)$ is decreasing, and so is $\hat{x}_{i}\left(X_{-i}\right)$.

We demonstrate now that the slope of the best response is (piecewise) lower than 1 , in absolute value. As the proof of Theorem 1 established, this property holds for its branches $x^{(1)}$ and $x^{(2)}$. We simply need to prove it for $x^{(3)}$. As the own and cross second order derivatives of the expected profit are both negative under Assumption 1 at the best response, $-A(X) x_{i}-$ $2 B(X)>0$ and $-A(X) x_{i}-\tilde{B}(X)>0$, and $\frac{d x_{i}^{(3)}}{d X_{-i}}<1$ in absolute value if

$$
\begin{aligned}
& \frac{\left|A(X) x_{i}+\tilde{B}(X)\right|}{\left|A(X) x_{i}+2 B(X)\right|}<1 \Leftrightarrow \tilde{B}(X)-2 B(X)>0 \\
& \Leftrightarrow-(1-F(X))+\frac{n^{2}+n+2}{(n+1)^{2}}(1-F(c+X))+2(1-F(X))-2 \frac{n^{2}+n+1}{(n+1)^{2}}(1-F(X+c))>0 \\
& \Leftrightarrow(1-F(X))-\frac{n}{n+1}(1-F(X+c))>0 \quad(59)
\end{aligned}
$$

which holds as $1-F(X)>1-F(X+c)$ and $\frac{n}{n+1}<1$. Consequently $x^{(3)}\left(X_{-i}\right)$ has a slope smaller than 1 in absolute value.

To conclude, under Assumption 1, the best response $\hat{x}_{i}\left(X_{-i}\right)$ is continuous and decreasing. Hence there exists a unique $x^{*}$ solution to $x^{*}=\hat{x}_{i}\left((n-1) x^{*}\right)$, which can be on one of the three branches of the best response depending on the model parameters. It suffices to study the intersection between the best response $\hat{x}_{i}\left(X_{-i}\right)$ and the line $x_{i}=X_{-i} /(n-1)$ in the graph $\left(X_{-i}, x_{i}\right)$, that is the solutions in $x^{*}$ to $x^{*}=\hat{x}_{i}\left((n-1) x^{*}\right)$. As established in the proof of Theorem 1, the equilibrium individual level of advance sales is given by $x_{I}^{*}$ if $a_{L}>\hat{a}$, where $\hat{a}$ is defined in (45). When this condition is not satisfied, the unique equilibrium level of advanced sales is given by either $x_{I I}^{*}$, the solution in $x$ of $\frac{\partial \pi_{I I}^{e}(x,(n-1) x)}{\partial x_{i}}=0$, or by $x_{I I I}^{*}$ the solution in $x$ of $\frac{\partial \pi_{I I I}^{e}(x,(n-1) x)}{\partial x_{i}}=0$.

\section{Proof of Corollary 3}

To demonstrate the corollary, it suffices to demonstrate that $(n-1) x_{I I}^{*}$ and $\frac{N\left(a_{L}\right)}{D\left(a_{L}\right)}$ (defined in (47)) intersect as $a_{L}$ changes, and that $(n-1) x_{I I}^{*}<\frac{N\left(a_{L}\right)}{D\left(a_{L}\right)}$ for large values of $a_{L}$, case in which the equilibrium advance sales are $x_{I I}^{*}$, while $(n-1) x_{I I}^{*}>\frac{N\left(a_{L}\right)}{D\left(a_{L}\right)}$ for small values of $a_{L}$, case in which the equilibrium advance sales are $x_{I I I}^{*}$. As $a_{L}$ varies between $c$ and $a_{H}-c$, let us compare $(n-1) x_{I I}^{*}$ and $\frac{N\left(a_{L}\right)}{D\left(a_{L}\right)}$ for these two extreme values of $a_{L}$.

By definition, $x_{I I}^{*}$ cannot be negative even if $a_{L}=c$ : indeed the marginal profit (19) evaluated at $(0,0)$ is a weighted average of differences between the demand intercept and the marginal cost $c$, which is strictly positive. Let us study the value of $N\left(a_{L}\right) / D\left(a_{L}\right)$ at the smallest 
possible value for $a_{L}$ we assume, $a_{L}=c$. We have

$$
\begin{gathered}
N(c)=2\left(\frac{\left((n+1)^{2}-n\right) F(2 c)+n}{(n+1)^{2}}\right) c-F(2 c)(E(a \mid a \leq 2 c)-c) \\
-\frac{(n-1)}{(n+1)^{2}}(1-F(2 c))(E(a \mid a \geq 2 c)-c) \\
=3 F(2 c) c-F(2 c)(E(a \mid a \leq 2 c) \\
+\frac{(3 n-1)}{(n+1)^{2}}(1-F(2 c)) c-\frac{(n-1)}{(n+1)^{2}}(1-F(2 c)) E(a \mid a \geq 2 c)
\end{gathered}
$$

which is negative if $c$ is sufficiently small. Hence $(n-1) x_{I I}^{*}>\frac{N\left(a_{L}\right)}{D\left(a_{L}\right)}$ for $a_{L}=c$ and $c$ sufficiently small and the equilibrium is of Type III.

Similarly $N\left(a_{H}-c\right) / D\left(a_{H}-c\right)=2\left(a_{H}-c\right)-\int_{a_{H}-c}^{a_{H}} a d F(a)+c>a_{H}-c$, which is a strictly dominated level of advance sales for all firms, and in particular larger than $(n-1) x_{I I}^{*}$ and $(n-1) x_{I I I}^{*}$. Hence $(n-1) x_{I I}^{*}<\frac{N\left(a_{L}\right)}{D\left(a_{L}\right)}$ for $a_{L}=a_{H}-c$ and the equilibrium is of Type II.

As $x_{I I}^{*}, N\left(a_{L}\right)$ and $D\left(a_{L}\right)$ are continuous in $a_{L}$, this is sufficient to ensure that $(n-1) x_{I I}^{*}$ and $\frac{N\left(a_{L}\right)}{D\left(a_{L}\right)}$ cross at least once for a particular value of $a_{L}$ if $c$ is sufficiently small, and that the equilibrium advance sales are $x_{I I}^{*}$ for $a_{L}$ large enough while it is $x_{I I I}^{*}$ for $a_{L}$ small enough. Let us denote the upper threshold value $\tilde{a}^{\prime \prime}$ at which both curves cross, and $\tilde{a}^{\prime}$ the lower threshold value (both can be identical to each other for many distributions $F(a)$, but we cannot rule out the theoretical possibility that $\tilde{a}^{\prime}<\tilde{a}^{\prime \prime}$ with multiple thresholds). Then from the argument above, $(n-1) x_{I I}^{*}<\frac{N\left(a_{L}\right)}{D\left(a_{L}\right)}$ for $a_{L}>\tilde{a}^{\prime \prime}$ and $(n-1) x_{I I}^{*}>\frac{N\left(a_{L}\right)}{D\left(a_{L}\right)}$ for $a_{L}<\tilde{a}^{\prime}$. 


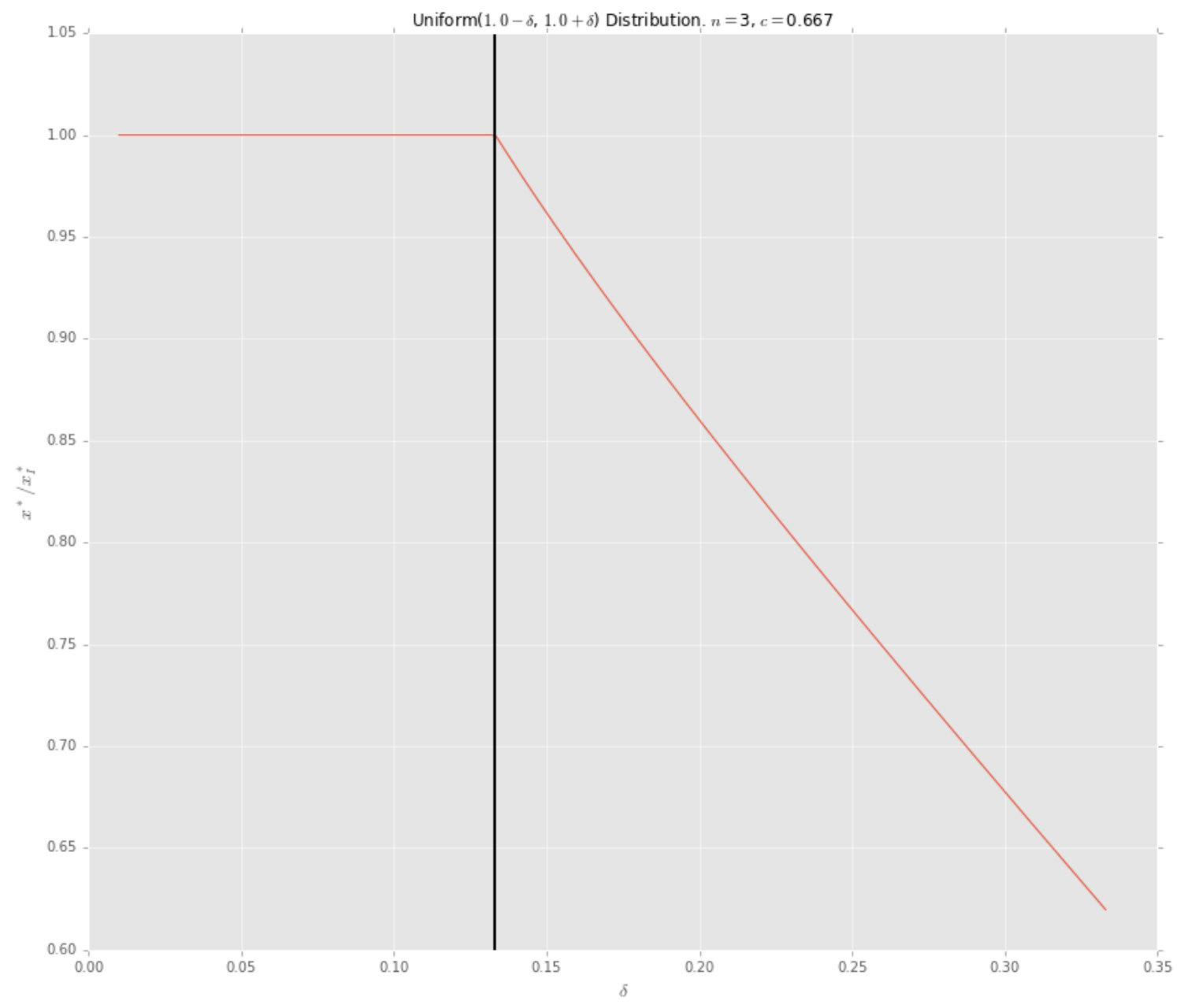

Figure 1: Equilibrium advance sales relative to $x_{I}^{*}$ 


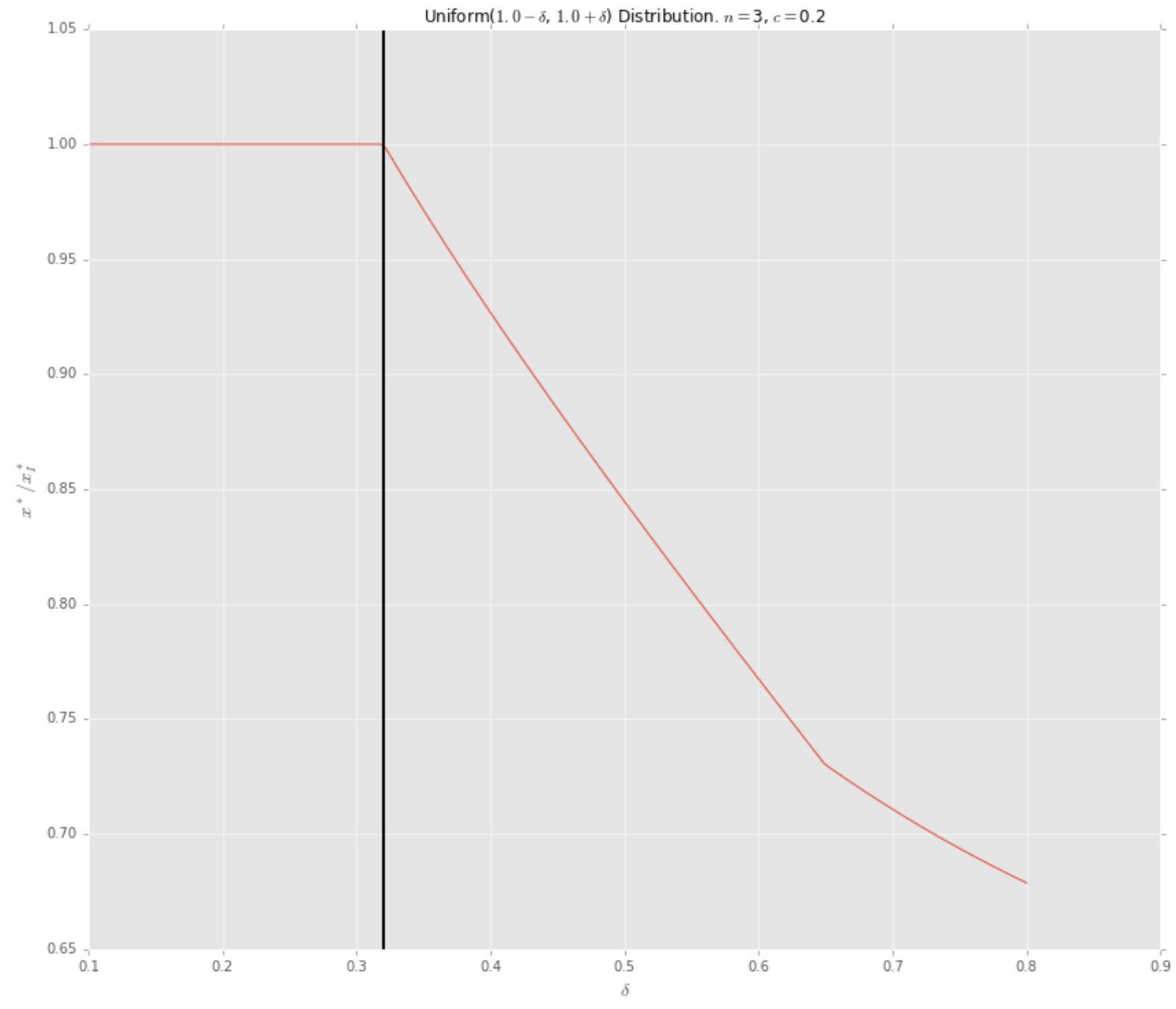

Figure 2: Equilibrium advance sales relative to $x_{I}^{*}$ 
Lognormal Distribution. $s=1.0, a_{L}=10, a_{H}=\infty, n=3, c=2.0$
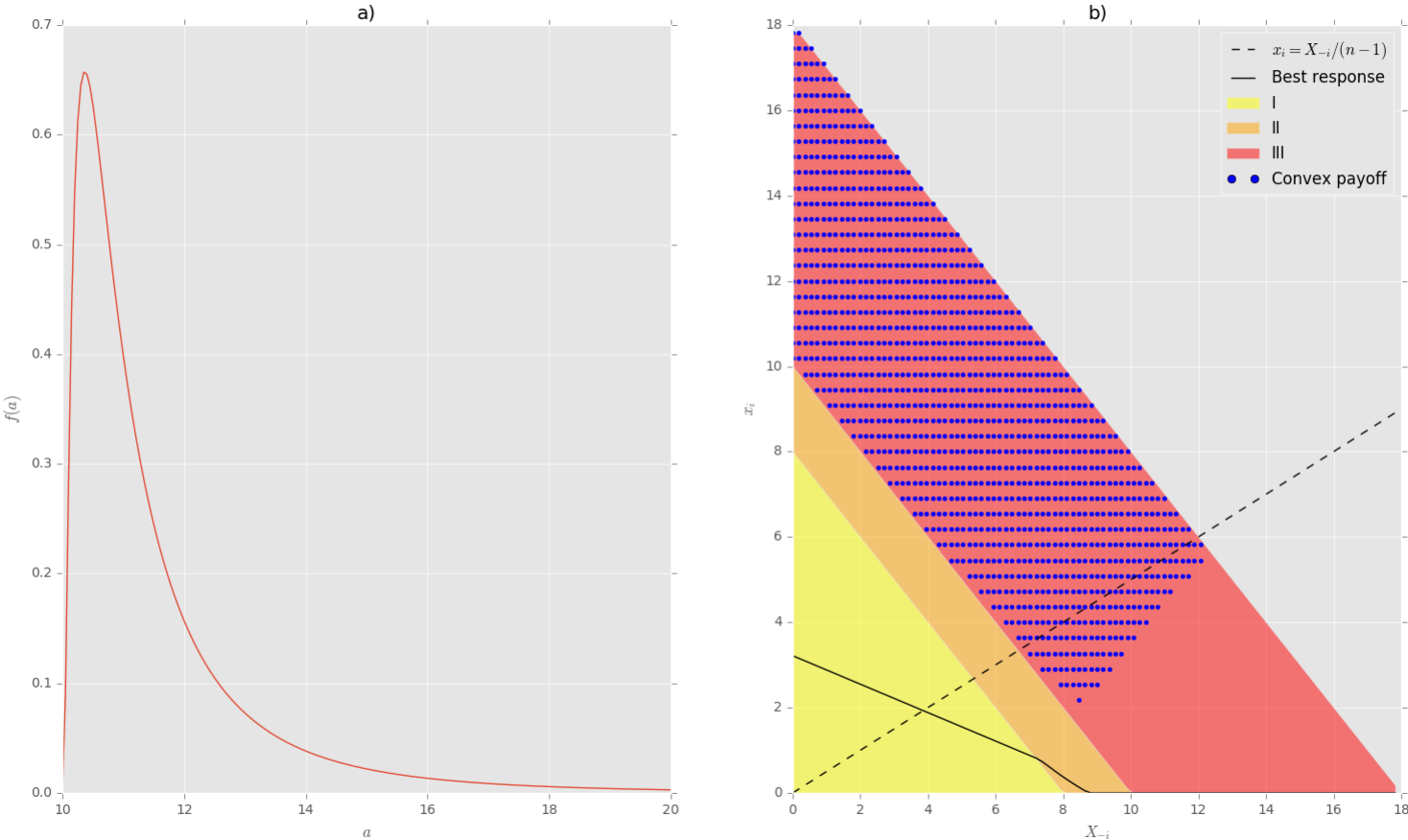

Figure 3: Lognormal distribution
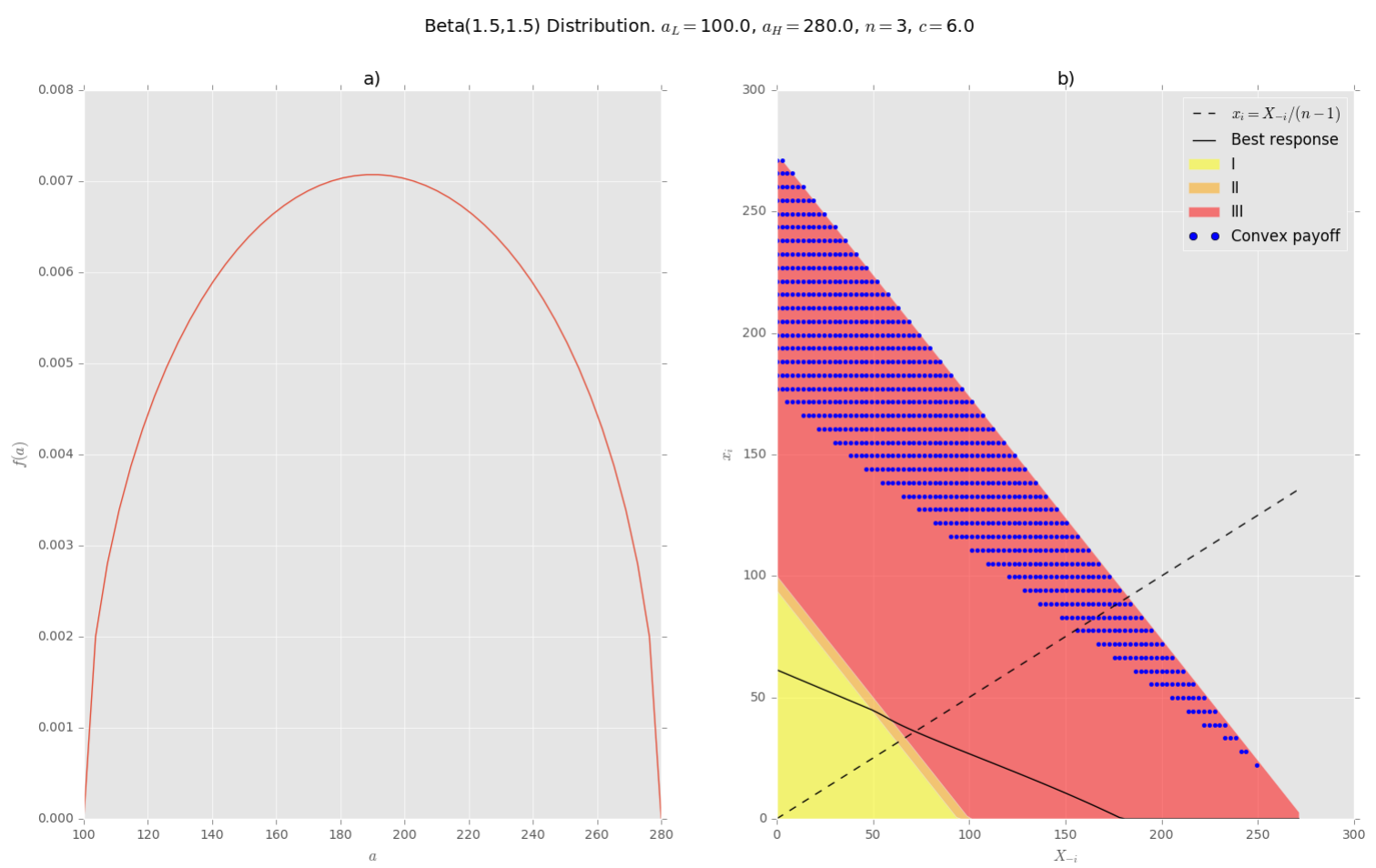

Figure 4: Best response: Symmetric, uni-modal distribution of demand 
$\operatorname{Beta}(0.5,0.5)$ Distribution. $a_{L}=100.0, a_{H}=280.0, n=3, c=6.0$

a)

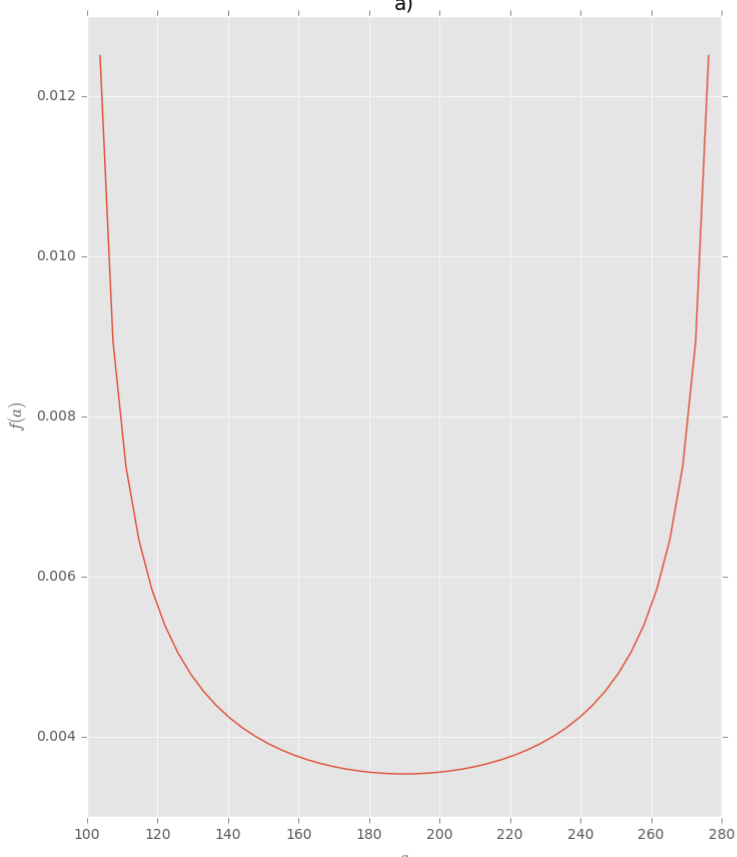

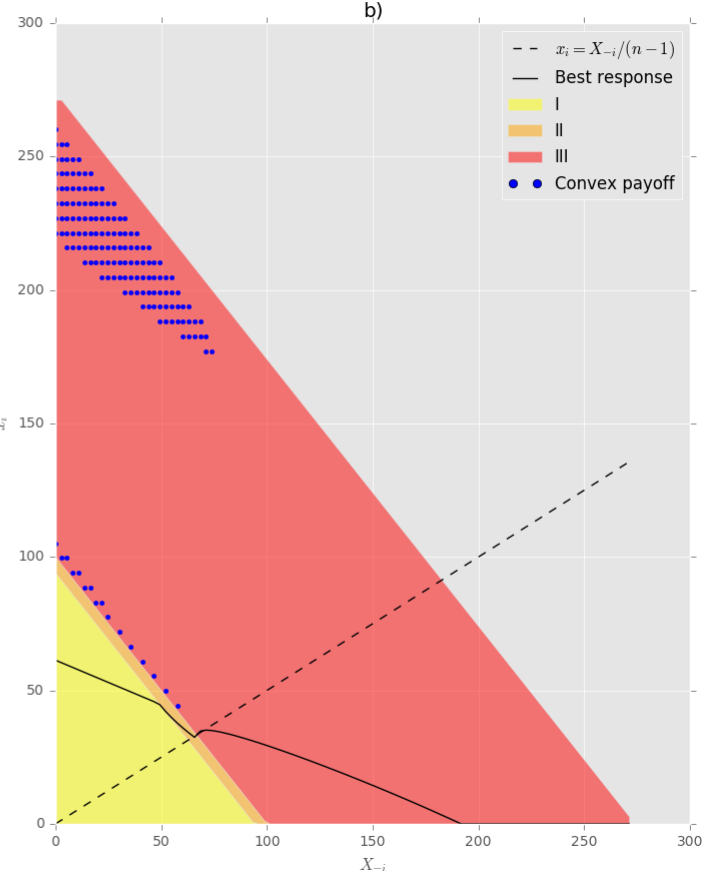

Figure 5: Best response: U-shaped distribution of demand
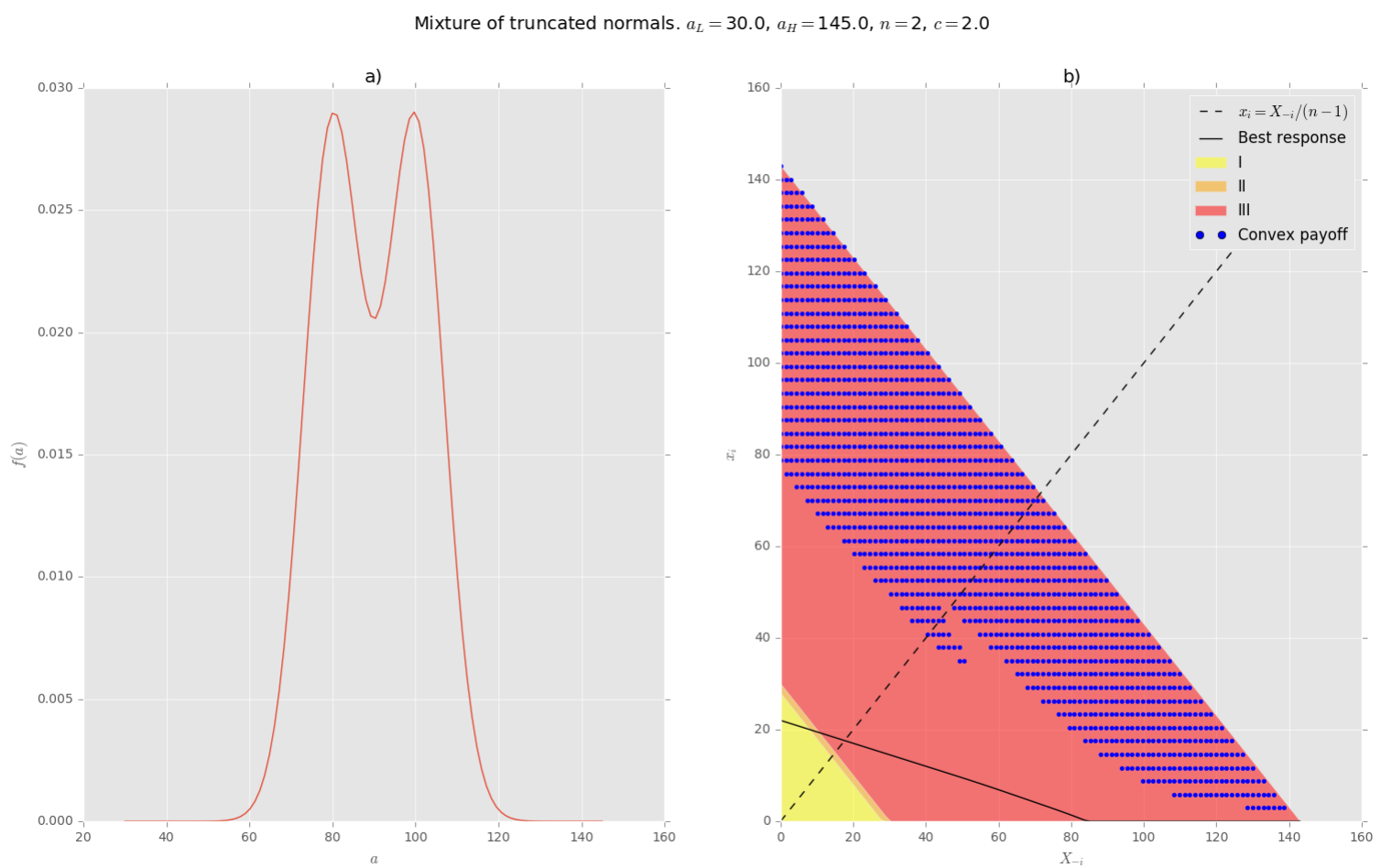

Figure 6: Bi-modal mixture with modes near together 
Mixture of truncated normals. $a_{L}=30.0, a_{H}=145.0, n=2, c=2.0$

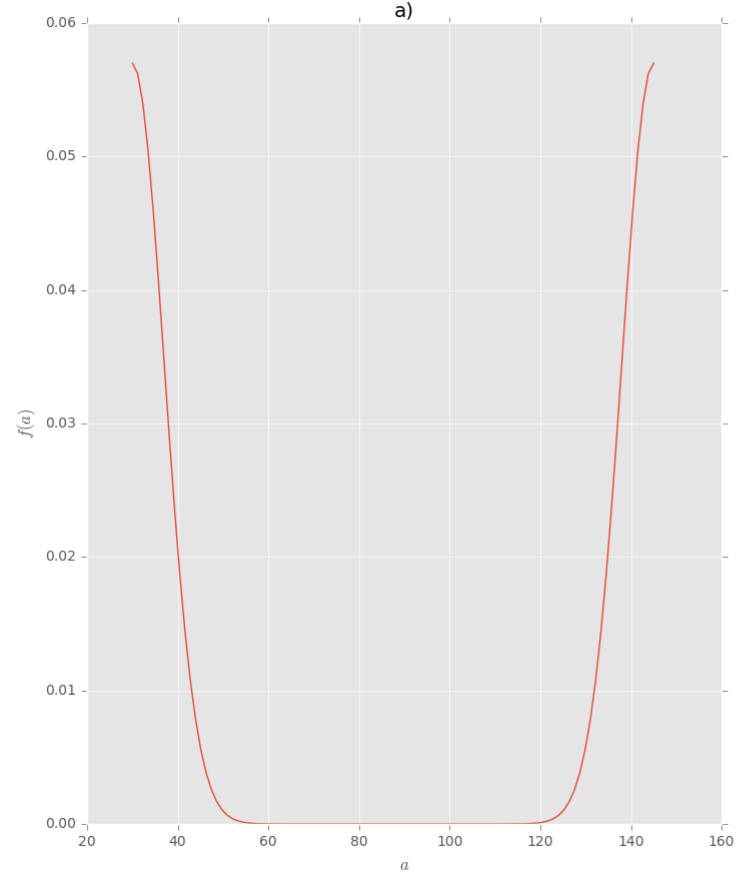

b)

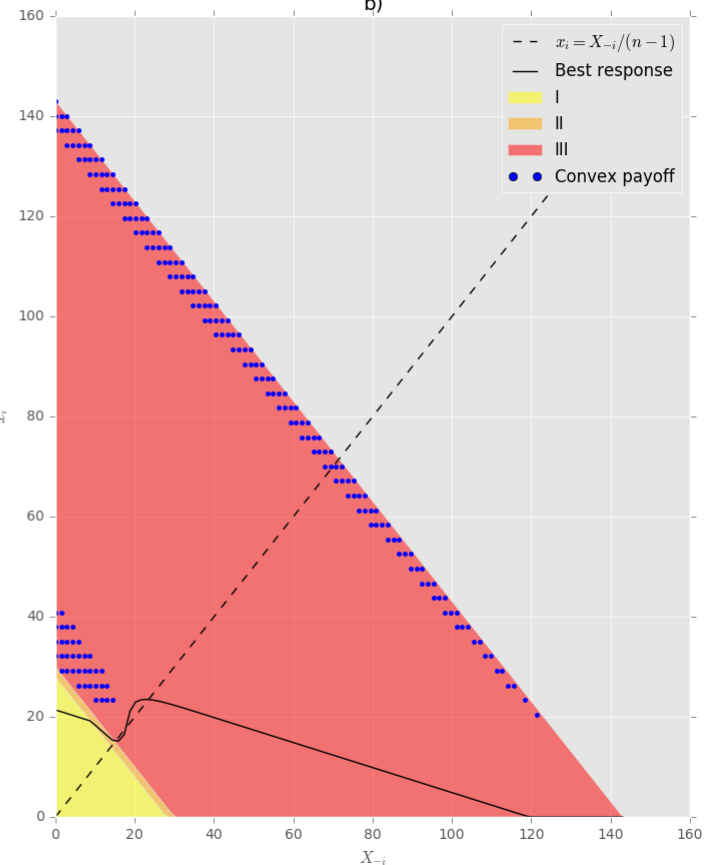

Figure 7: A bi-modal mixture with modes at bounds 